\title{
Local and CNS-Wide Astrocyte Intracellular Calcium Signaling Attenuation In Vivo with CalEx ${ }^{\text {flox }}$ Mice
}

\author{
${ }^{1}$ Xinzhu Yu, ${ }^{1,3}$ Stefanie L. Moye, ${ }^{1}$ and Baljit S. Khakh ${ }^{1,2}$ \\ ${ }^{1}$ Department of Physiology, ${ }^{2}$ Department of Neurobiology, David Geffen School of Medicine, University of California Los Angeles, Los Angeles, \\ California 90095-1751, and ${ }^{3}$ Department of Molecular and Integrative Physiology, Beckman Institute, University of Illinois at Urbana-Champaign, \\ Urbana, Illinois 61801-3704
}

Astrocytes exist throughout the CNS and affect neural circuits and behavior through intracellular $\mathrm{Ca}^{2+}$ signaling. Studying the function(s) of astrocyte $\mathrm{Ca}^{2+}$ signaling has proven difficult because of the paucity of tools to achieve selective attenuation. Based on recent studies, we generated and used male and female knock-in mice for Cre-dependent expression of mCherrytagged hPMCA2w/b to attenuate astrocyte $\mathrm{Ca}^{2+}$ signaling in genetically defined cells in vivo (CalEx ${ }^{\text {flox }}$ mice for Calcium Extrusion). We characterized CalEx ${ }^{\text {flox }}$ mice following local AAV-Cre microinjections into the striatum and found reduced astrocyte $\mathrm{Ca}^{2+}$ signaling ( $\left.90 \%\right)$ accompanied with repetitive self-grooming behavior. We also crossed CalEx ${ }^{\text {flox }}$ mice to astrocyte-specific Aldh1l1-Cre/ERT2 mice to achieve inducible global CNS-wide $\mathrm{Ca}^{2+}$ signaling attenuation. Within $6 \mathrm{~d}$ of induction in the bigenic mice, we observed significantly altered ambulation in the open field, disrupted motor coordination and gait, and premature lethality. Furthermore, with histologic, imaging, and transcriptomic analyses, we identified cellular and molecular alterations in the cerebellum following mCherry-tagged hPMCA2w/b expression. Our data show that expression of mCherry-tagged hPMCA2w/b with CalEx ${ }^{\text {flox }}$ mice throughout the CNS resulted in substantial attenuation of astrocyte $\mathrm{Ca}^{2+}$ signaling and significant behavioral alterations in adult mice. We interpreted these findings candidly in relation to the ability of CalEx to attenuate astrocyte $\mathrm{Ca}^{2+}$ signaling, with regards to additional mechanistic interpretations of the data, and their relation to past studies that reduced astrocyte $\mathrm{Ca}^{2+}$ signaling throughout the CNS. The data and resources provide complementary ways to interrogate the function(s) of astrocytes in multiple experimental scenarios.

Key words: Aldh111-Cre/ERT2; astrocyte; calcium; striatum

Significance Statement

Astrocytes represent a significant fraction of all brain cells and tile the entire central nervous system. Unlike neurons, astrocytes lack propagated electrical signals. Instead, astrocytes are proposed to use diverse and dynamic intracellular $\mathrm{Ca}^{2+}$ signals $^{2}$ to communicate with other cells. An open question concerns if and how astrocyte $\mathrm{Ca}^{2+}$ signaling regulates behavior in adult mice. We approached this problem by generating a new transgenic mouse line to achieve inducible astrocyte $\mathrm{Ca}^{2+}$ signaling attenuation in vivo. We report our data with this mouse line and we interpret the findings candidly in relation to past studies and within the framework of different mechanistic interpretations.

Received Jan. 13, 2021; revised Mar. 21, 2021; accepted Mar. 30, 2021.

Author contributions: X.Y. and B.S.K. designed research; X.Y. and S.L.M. performed research; X.Y. and S.L.M. analyzed data; X.Y. and B.S.K. wrote the paper.

S.L.M. and X.Y. were supported by National Institutes of Health Grants NS111583 and MH104069 and an Allen Distinguished Investigator Award through The Paul G. Allen Frontiers Group. The Khakh lab was supported by the Ressler Family Foundation. This work was also supported by the National Institute of Neurological Disorders and Stroke Informatics Center for Neurogenetics and Neurogenomics Grant P30 NS062691 and by the Genetics, Genomics, and Informatics Core of the Semel Institute of Neuroscience at UCLA Grant U54HD087101-01 from the Eunice Kennedy Shriver National Institute of Child Health and Human Development. We thank the University of California Los Angeles (UCLA) Neuroscience Genomics Core for assistance with sequencing and Fuying Gao and Giovanni Coppola for helping with RNA-seq data analysis. We also thank Marsa Taheri for installing AQuA on the lab computer, showing others how to use it, for help with some analysis, and for comments on a previous version of this manuscript.

The authors declare no competing financial interests.

Correspondence should be addressed to Baljit S. Khakh at bkhakh@mednet.ucla.edu or Xinzhu Yu at xinzhuyu@illinois.edu.

https://doi.org/10.1523/JNEUROSCI.0085-21.2021

Copyright $@ 2021$ the authors

\section{Introduction}

Important progress has been made exploring astrocytes, especially their contributions to synapse formation and removal (Allen and Lyons, 2018). However, the field lacks comprehensive understanding of how astrocytes perform multiple functions to regulate the cells they contact, and how they influence neural circuits and behavior. One hindrance has been the lack of tools with which to attenuate or activate astrocyte signaling within the adult CNS (Hirbec et al., 2020; Yu et al., 2020a).

Astrocytes display intracellular $\mathrm{Ca}^{2+}$ signaling dynamics, which are well documented and proposed to underlie physiological functions (Howarth, 2014; Volterra et al., 2014; Oliveira et al., 2015; Bazargani and Attwell, 2016; Shigetomi et al., 2016; Verkhratsky and Nedergaard, 2018; Yu et al., 2020a; Nagai et al., 
2021). The question of whether astrocyte $\mathrm{Ca}^{2+}$ signals are causal for changes in neural circuit function and behavior in adult mice is beginning to be directly explored. Such experiments have become possible because of reliable methods to target astrocytes genetically (Yu et al., 2020a) and to monitor astrocyte $\mathrm{Ca}^{2+}$ signaling with genetically encoded calcium indicators (Hires et al., 2008; Russell, 2011; Volterra et al., 2014; Nimmerjahn and Bergles, 2015; Shigetomi et al., 2016). These advances have, in turn, necessitated the use of methods to both increase and attenuate $\mathrm{Ca}^{2+}$ signals within astrocytes in vivo and to explore the consequences. A variety of methods now exist to increase astrocyte $\mathrm{Ca}^{2+}$ signaling, including LiGluR, channelrhodopsinbased effectors, melanopsin, optoXRs, and designer receptors exclusively activated by designer drugs (Hirbec et al., 2020; Yu et al., 2020a). In contrast, despite progress with $\mathrm{IP}_{3}$ receptor type 2 deletion mice $\left(\mathrm{IP}_{3} \mathrm{R} 2-/-\right)$ and $\mathrm{IP}_{3}$ sponges (Petravicz et al., 2008; Agulhon et al., 2010; Xie et al., 2010), the development of approaches to efficiently attenuate astrocyte $\mathrm{Ca}^{2+}$ signaling has lagged behind, probably because of the diverse nature of astrocyte $\mathrm{Ca}^{2+}$ signaling. Furthermore, although the use of $\mathrm{IP}_{3} \mathrm{R} 2-/-$ mice initially furnished little evidence for a role for astrocyte $\mathrm{IP}_{3^{-}}$ mediated $\mathrm{Ca}^{2+}$ signals in regulating behavior, subsequent studies have found that such mice display altered responses in a variety of settings (see Fiacco and McCarthy, 2018; Savtchouk and Volterra, 2018). The realization that $\mathrm{IP}_{3} \mathrm{R} 2-/-$ mice show astrocyte $\mathrm{Ca}^{2+}$ signaling that was undetected in earlier studies has also emphasized the need for additional methods to attenuate astrocyte $\mathrm{Ca}^{2+}$ signaling to explore physiological functions (Di Castro et al., 2011; Srinivasan et al., 2015; Rungta et al., 2016; Agarwal et al., 2017).

Recently, we developed a genetic approach to attenuate endogenous astrocyte $\mathrm{Ca}^{2+}$ signaling in vivo regardless of the $\mathrm{Ca}^{2+}$ source (Yu et al., 2018). A human plasma membrane $\mathrm{Ca}^{2+}$ ATPase pump (type 2, variant w/b; hPMCA2w/b) that constitutively extrudes $\mathrm{Ca}^{2+}$ from cells and which is not endogenously expressed in astrocytes was used. $G f a A B C_{1} D$ adeno-associated virus 2/5 (AAV2/5)-mediated overexpression of hPMCA2w/b on the plasma membrane of astrocytes in the striatum reduced spontaneous and G-protein-coupled receptor (GPCR)-mediated $\mathrm{Ca}^{2+}$ signals, resulting in enhanced repetitive behaviors ( $\mathrm{Yu}$ et al., 2018). We termed the hPMCA2w/b approach CalEx, a mnemonic for $\mathrm{Ca}^{2+}$ extrusion. To further improve CalEx, we developed knock-in mice at the Rosa26 locus (CalEx ${ }^{\text {flox }}$ mice) to potentially enable both circuit-specific and global CNS-wide $\mathrm{Ca}^{2+}$ attenuation in vivo. hPMCA2w/b expression was controlled by Cre expression and resulted in more rapid and greater $\mathrm{Ca}^{2+}$ signaling attenuation than previously possible (Yu et al., 2018). We employed CalEx ${ }^{\text {flox }}$ mice to explore the consequences of CNS-wide attenuation of astrocyte $\mathrm{Ca}^{2+}$ signaling, and based on the empirical data we performed gene expression analyses to report likely molecular mechanisms. Our studies show that CalEx ${ }^{\text {flox }}$ mice can be used to efficiently attenuate astrocyte $\mathrm{Ca}^{2+}$ signaling. Thus, we provide well-characterized resources to broadly explore astrocyte signaling in vivo in multiple experimental scenarios. We also carefully consider mechanistic interpretations of our findings in relation to past studies on the topic and discuss future uses, caveats, and further developments.

\section{Materials and Methods}

\section{Material availability}

All unique/stable reagents generated in this study are available on request. Line $1 \mathrm{CalEx}^{\text {flox }}$ mice have been deposited at JAX with genetic

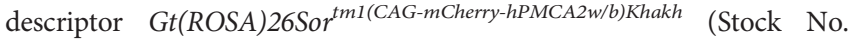
035252). RNA-seq data have been deposited at the Gene Expression Omnibus (GEO; \#GSE164235).

\section{Data availability}

All data are available on request from the corresponding author.

\section{Mouse models}

All animal experiments were conducted in accordance with the National Institutes of Health Guide for the Care and Use of Laboratory Animals and were approved by the Chancellor's Animal Research Committee at the University of California, Los Angeles (UCLA). Male and female mice aged between 6 and 12 weeks old were used in this study. Mice were housed in the vivarium managed by the Division of Laboratory Animal Medicine at UCLA with a 12/12 h light/dark cycle and ad libitum access to food and water. Wild-type C57BL/6NTac and Aldh1l1-Cre/ERT2 mice were generated from in house breeding colonies. CalEx ${ }^{\text {flox }}$ mice were backcrossed with C57BL/6NTac for more than eight generations. Heterozygous CalEx ${ }^{\text {flox }}$ mice were crossed with Aldh1l1-Cre/ERT2 mice (Srinivasan et al., 2016) to generate bigenic mice carrying both alleles.

\section{Generation of CalEx ${ }^{\text {flox }}$ mice at the Rosa26 locus}

The Rosa26 targeting plasmid was modified from the Ai39 plasmid (Addgene \#34884) to contain transgene mCherry-hPMCA2w/b and the knock-in mice were generated using well-established methods (Srinivasan et al., 2016). The $\mathrm{CalEx}^{\text {flox }}$ mice contain a CMV-IE enhancer/chicken $\beta$-actin/rabbit $\beta$-globin hybrid promoter (CAG) promoter, followed by a loxP-3 $\times$ SV40pA-loxP cassette and the mCherryhPMCA2w/b cDNA at the Rosa26 locus. Briefly, CalEx transgene was amplified by PCR from the pZac2.1-G $f a A B C_{1} D$-mCherry-hPMCA2w/b plasmid (Addgene \#111568) and subcloned between MluI sites in the Ai39 plasmid to create the CalEx Rosa26 targeting plasmid. All cloning was performed using Stbl2 cells (Invitrogen) that were propagated at $30^{\circ}$ $\mathrm{C}$ to prevent recombination. Sequences of the targeting construct were confirmed with diagnostic restriction digests, PCR amplification and sequencing of the ampicillin and neomycin resistance cassette as well as the $5^{\prime}$ and $3^{\prime}$ homology arms. For electroporation of embryonic stem (ES) cells, the CalEx Rosa26 targeting plasmid was linearized with AclI, which cuts at two sites within the ampicillin resistance cassette, and then purified with a concentration of $1015 \mathrm{ng} / \mu \mathrm{l}$. Linearized plasmid DNA was electroporated into C57BL/6 JM8.N4 ES cells and 288 clones were picked to expand in 96-well plates at the UC Davis Mouse Biology Program (MBP). Recombinant clones were then injected into blastocysts from $\mathrm{BALB} / \mathrm{cAnNTac}$ donors. Chimeric animals were scored based on coat color and animals that showed $>50 \%$ chimerism were bred with wild-type mice to confirm germline transmission. Heterozygous CalEx ${ }^{\text {flox }}$ mice were backcrossed with C57BL/6NTac mice for more than eight generations, i.e., to $\sim 99.6 \% \mathrm{C} 57 \mathrm{BL} / 6 \mathrm{NTac}$. Both heterozygous and homozygous $\mathrm{CalEx}{ }^{\text {flox }}$ mice are viable and fertile. All the results presented in this study were generated with heterozygous $\mathrm{CalEx}^{\text {flox }}$ mice. Genotypes of CalEx ${ }^{\text {flox }}$ mice were performed by PCR with genomic DNA from either the ear or tail biopsies. Wild-type allele was confirmed by forward primer $5^{\prime}$-CCTTTCTGGGAGTTCTCTGCTGC-3'; reverse primer 5'-GCGGATCACAAGCAATAATAACCTG-3'; giving a 332 bp amplicon. CalEx flox allele was confirmed by forward primer $5^{\prime}$ CCTTTCTGGGAGTTCTCTGCTGC-3'; reverse primer $5^{\prime}$-CGTA AGTTATGTAACGCGGAACTCC-3'; giving a 217-bp amplicon.

\section{Generation of CalEx ${ }^{\text {flox }} \times$ Aldh1l1-Cre/ERT2 bigenic mice}

To generate bigenic mice, heterozygous $\mathrm{CalEx}^{\text {flox }}$ mice were crossed with hemizygous Aldh1l1-Cre/ERT2 mice. Bigenic pups were identified by genotyping for CalEx insert at the ROSA26 locus (forward primer $5^{\prime}$ CCTTTCTGGGAGTTCTCTGCTGC-3'; reverse primer $5^{\prime}$-CGTAA GTTATGTAACGCGGAACTCC-3') and Cre/ERT2 (forward primer 5'-AGACCAATCATCAGGATCTCTAGCC-3'; reverse primer $5^{\prime}$ CATGCAAGCTGGTGGCTGG-3') in two separate PCR reactions for each pup. Bigenic mice used for experiments were heterozygous for CalEx at the ROSA26 locus and hemizygous for the Aldh1l1-Cre/ERT2 insert (CalEx ${ }^{\text {flox/wt }}$; Aldh1l1-Cre/ERT2). Three littermate control groups 
were included in all the experiments: (1) wild-type mice; (2) Aldh1l1Cre/ERT2 mice; and (3) CalEx ${ }^{\text {flox/wt }}$ mice.

\section{Tamoxifen administration}

Tamoxifen (Sigma, T5648) was freshly prepared at a concentration of $25 \mathrm{mg} / \mathrm{ml}$ in corn oil and dissolved at $37^{\circ} \mathrm{C}$ with continuous agitation. As previously described (Srinivasan et al., 2016), to induce gene expression, tamoxifen was administered intraperitoneally at $75 \mathrm{mg} / \mathrm{kg}$ body weight for $1 \mathrm{~d}$ or five consecutive days, using a 1 -ml syringe and a $25-\mathrm{G} 5 / 8$ inch needle (BD Biosciences).

\section{Surgical procedure of in vivo microinjection}

Surgical procedures for viral microinjections have been described previously (Shigetomi et al., 2013; Chai et al., 2017; Yu et al., 2018; Nagai et al., 2019). In brief, mice aged six to nine weeks old were anesthetized and placed onto a stereotaxic frame (David Kopf Instruments, Tujunga $\mathrm{CA})$. Continuous anesthesia using isoflurane was carefully monitored and adjusted throughout the surgery. Mice were injected with buprenorphine (Buprenex; $0.1 \mathrm{mg} / \mathrm{kg}$ ) subcutaneously before surgery. Scalp incisions were made and craniotomies $(\sim 1 \mathrm{~mm}$ in diameter) were created using a high-speed drill (Henry Schein Medical Dental) for unilateral viral injections while two craniotomies were made above both parietal cortices for bilateral viral injections. Beveled glass pipettes (1B100-4; World Precision Instruments) filled with viruses were placed into the dorsal striatum $(0.8 \mathrm{~mm}$ anterior to the bregma, $2.0 \mathrm{~mm}$ lateral to the midline, and $2.4 \mathrm{~mm}$ from the pial surface) or the hippocampal CA1 $(2.0 \mathrm{~mm}$ posterior to the bregma, $1.5 \mathrm{~mm}$ lateral to the midline, and $1.6 \mathrm{~mm}$ from the pial surface) or the cerebellum ( $6.0 \mathrm{~mm}$ posterior to the bregma, 1.5 $\mathrm{mm}$ lateral to the midline, and $1.0 \mathrm{~mm}$ from the pial surface). AAVs were injected at $200 \mathrm{nl} / \mathrm{min}$ using a microinjection syringe pump (UMP3; World Precision Instruments). Glass pipettes were withdrawn after $10 \mathrm{~min}$, and scalps were cleaned and sutured with sterile surgical sutures. Mice were allowed to recover in clean cages with food containing trimethoprim/sulfamethoxazole and water for $7 \mathrm{~d}$. Subsequent histologic, imaging and behavioral experiments were performed 3-21 d after surgeries.

Viruses used in this study included: AAV2/5 $G f a A B C_{1} D$-Cre, AAV2/ $5 \mathrm{GfaABC} C_{1} D$-GCaMP6f, and AAV2/5 $G f a A B C_{1} D$-tdTomato. All of these have been previously characterized for the striatum at the ages used in this study (Tong et al., 2014; Jiang et al., 2016; Chai et al., 2017; Octeau et al., 2018; Yu et al., 2018; Nagai et al., 2019). Viruses were injected with a total volume of $0.2-0.5 \mu \mathrm{l}$ per site to deliver $\sim 10^{10}$ genome copies into the dorsal striatum or the hippocampal CA1 or the cerebellum.

\section{Brain slice preparation for $\mathrm{Ca}^{2+}$ imaging}

Mice were transferred from the vivarium to the laboratory during the light cycle between 8 and 10 A.M. Brain slices were prepared 30 min to 1 $\mathrm{h}$ afterward and were used for experiments within $8 \mathrm{~h}$ of slicing (mostly within $6 \mathrm{~h}$ ). Both male and female mice (8-11 weeks old) were anesthetized and decapitated approximately two to four weeks after AAV injection. Coronal slices $(300 \mu \mathrm{m}$ thick) of the striatum or sagittal slices (300 $\mu \mathrm{m}$ thick) of the cerebellum were prepared in ice-cold sucrose cutting solution $\left(30 \mathrm{~mm} \mathrm{NaCl}, 4.5 \mathrm{~mm} \mathrm{KCl}, 1.2 \mathrm{~mm} \mathrm{NaH}_{2} \mathrm{PO}_{4}, 26 \mathrm{~mm}\right.$ $\mathrm{NaHCO}_{3}, 10 \mathrm{~mm}$ D-glucose, $194 \mathrm{~mm}$ sucrose, and $1 \mathrm{~mm} \mathrm{MgCl}_{2}$ ) using a vibratome (DSK Microslicer; Ted Pella). Slices were then incubated in artificial ACF (ACSF; $124 \mathrm{~mm} \mathrm{NaCl}, 4.5 \mathrm{~mm} \mathrm{KCl}, 1 \mathrm{~mm} \mathrm{MgCl}_{2}, 1.2 \mathrm{~mm}$ $\mathrm{NaH}_{2} \mathrm{PO}_{4}, 26 \mathrm{~mm} \mathrm{NaHCO}, 10 \mathrm{~mm}$ D-glucose, and $2.0 \mathrm{~mm} \mathrm{CaCl}_{2}$ ) for $30 \mathrm{~min}$ at $32^{\circ} \mathrm{C}$ and then $1 \mathrm{~h}$ at room temperature before recording. All the solutions were oxygenated with $95 \% \mathrm{O}_{2} / 5 \% \mathrm{CO}_{2}$.

\section{Intracellular $\mathrm{Ca}^{2+}$ imaging of astrocytes and analysis}

$\mathrm{Ca}^{2+}$ imaging in brain slices was performed using a Scientifica two-photon laser-scanning microscope equipped with a MaiTai laser (SpectraPhysics). To image GCaMP6f signals, the laser was tuned at 920-nm wavelength. The laser power measured at the sample was $<30 \mathrm{~mW}$ with a $40 \times$ water-immersion objective lens (Olympus). Striatal astrocytes or Bergmann glia located at least $40 \mu \mathrm{m}$ from the slice surface were selected for imaging. Images were acquired at one frame per second using SciScan software (Scientifica). Brain slices were maintained in ACSF
(124 mm NaCl, $4.5 \mathrm{~mm} \mathrm{KCl}, 1 \mathrm{~mm} \mathrm{MgCl}_{2}, 1.2 \mathrm{~mm} \mathrm{NaH}{ }_{2} \mathrm{PO}_{4}, 26 \mathrm{~mm}$ $\mathrm{NaHCO}_{3}, 10 \mathrm{~mm}$ D-glucose, and $2.0 \mathrm{mM} \mathrm{CaCl}_{2}$ ) through a perfusion system. Phenylephrine (PE; $10 \mu \mathrm{M})$ or ATP $(100 \mu \mathrm{M})$ was bath-applied for 2 min to activate GPCR-mediated $\mathrm{Ca}^{2+}$ signals. $0.25 \mu \mathrm{M}$ TTX was present throughout the experiment.

For region of interest (ROI)-based analysis, the somata, major branches and territories of striatal astrocytes and the somata of Bergmann glia were manually demarcated and $\mathrm{Ca}^{2+}$ signals recorded in a single optical plane were measured by plotting the intensity of regions of interest over time. Slow drifts in cell position $(\sim 2-5 \mu \mathrm{m})$ were corrected with a custom Plugin in NIH ImageJ. A signal was declared as a $\mathrm{Ca}^{2+}$ transient if it exceeded the baseline by greater than twice the baseline noise (SD). $\mathrm{Ca}^{2+}$ signals were processed in ImageJ and presented as the relative change in fluorescence $(\Delta \mathrm{F} / \mathrm{F})$. Peak amplitude, half-width, frequency and integrated areas of $\mathrm{Ca}^{2+}$ signals were analyzed in OriginPro 2016 (OriginLab).

For event-based analysis, 1-min recording of spontaneous $\mathrm{Ca}^{2+}$ signals was de noised in ImageJ and then analyzed with AQuA in MATLAB (Wang et al., 2019) to detect individual $\mathrm{Ca}^{2+}$ events. The AQuA version used was downloaded in December 2019 from Github. Parameters were adjusted for cytosolic GCaMP6f ex vivo experiments.

\section{Immunohistochemistry (IHC) and analysis}

Both male and female mice (6-12 weeks old) were transcardially perfused with 0.1 м PBS followed by $10 \%$ formalin. Brains were dissected out and postfixed in $10 \%$ formalin for several hours and then cryoprotected in $30 \%$ sucrose $(0.1 \mathrm{~m}$ PBS). Serial $40-\mu \mathrm{m}$ sections were collected and incubated with the following primary antibodies overnight at $4^{\circ} \mathrm{C}$ : mouse anti-S100 $\beta$ (1:1000; Sigma, S2532), mouse anti-NeuN (1:1000; Millipore, MAB377), and rabbit anti-RFP (1:1000; Rockland, 600-401379). The sections were then washed three times in $0.1 \mathrm{~m}$ PBS for $10 \mathrm{~min}$ each before incubation at room temperature for $2 \mathrm{~h}$ with secondary antibodies diluted in $0.1 \mathrm{~m}$ PBS. Alexa-conjugated (ThermoFisher Scientific) secondary antibodies were used at 1:1000 dilution. Fluorescent images were acquired with a $40 \times$ oil-immersion objective lens (NA 1.3) under a laser-scanning confocal microscope (FV1000; Olympus). Whole brain images were acquired with a $10 \times$ air objective lens (NA 0.3 ) on a confocal laser-scanning microscope (C2; Nikon). Laser settings were kept the same within each experiment. Images represent maximum intensity projections of optical sections with a step size of $1.0 \mu \mathrm{m}$. Images were processed with ImageJ. Cell counting was done on maximum intensity projections using the Cell Counter plugin; only cells with soma completely within the ROI were counted.

\section{Behavioral tests}

Behavioral tests were performed during the light cycle between 12 and 6 P.M. Both male and female mice (6-12 weeks old) were used in behavioral tests. All the experimental mice were transferred to the behavior testing room at least $30 \mathrm{~min}$ before the tests to acclimatize to the environment and to reduce stress. Temperature and humidity of the experimental rooms were kept at $23 \pm 2^{\circ} \mathrm{C}$ and $55 \pm 5 \%$, respectively. Background noise $(65 \pm 2 \mathrm{~dB})$ was generated by a white noise generator (San Diego Instruments).

\section{Open field test}

The open field chamber consisted of a square arena $(28.7 \times 30 \mathrm{~cm})$ enclosed by walls made of translucent polyethylene $(15 \mathrm{~cm}$ tall). The brightness of the experimental room was kept $<10$ lux. Locomotor activity of mice at 6-12 weeks old was then recorded for $10 \mathrm{~min}$ using an infrared camera located above the open field chamber. The open field behaviors were analyzed with an automated video tracking software ANY-maze from Stoelting.

\section{Self-grooming behavior}

Mice were placed individually into plastic cylinders $(15 \mathrm{~cm}$ in diameter and $35 \mathrm{~cm}$ tall) and allowed to habituate for $20 \mathrm{~min}$. The brightness of the experimental room was kept $<10$ lux. Self-grooming behavior was recorded for $10 \mathrm{~min}$. A timer was used to assess the cumulative time spent in self-grooming behavior, which included paw licking, unilateral 
and bilateral strokes around the nose, mouth, and face, paw movement over the head and behind ears, body fur licking, body scratching with hind paws, tail licking, and genital cleaning. The number of self-grooming bouts was also counted. Separate grooming bouts were considered when the pause was $>5 \mathrm{~s}$ or behaviors other than self-grooming occurred. Self-grooming microstructure was not assessed.

\section{Rotarod test}

Mice were held by the tails and placed on the rod $(3 \mathrm{~cm}$ diameter $)$ of a single lane rotarod apparatus (ENV-577 M, Med Associates Inc.), facing away from the direction of rotation. The rotarod was set with a start speed of $4 \mathrm{rpm}$. Acceleration started $10 \mathrm{~s}$ later and was set to $20 \mathrm{rpm} / \mathrm{min}$ with a maximum speed $40 \mathrm{rpm}$. Each mouse received two trials $30 \mathrm{~min}$ apart and the latency to fall was recorded for each trial. The average latency to fall was used as a measurement for motor coordination (Deacon, 2013).

\section{Footprint test}

A 1-m-long runway ( $8 \mathrm{~cm}$ wide) was lined with paper. Each mouse with hind paws painted with non-toxic ink was placed at an open end of the runway and allowed to walk to the other end with a darkened box. For the gait analysis, stride length and width were measured and averaged for both left and right hindlimbs over five steps.

\section{Cerebellar RNA-sequencing (RNA-seq) and analysis}

To perform bulk RNA-seq of the $\mathrm{Cb}, 5 \mathrm{~d}$ after the single dose tamoxifen injection $\left(75 \mathrm{mg} / \mathrm{kg}\right.$ body weight), cerebella from $\mathrm{CalEx}^{\text {flox/wt }}$ and bigenic mice were dissected out and frozen in a tissue transition solution (Invitrogen, \#AM7030) overnight. The tissues were homogenized in ice-cold lysis buffer and the cerebellar RNA was extracted using a mirVana miRNA isolation kit following the manufacturer's instruction (Invitrogen, \#AM1560). RNA concentration and quality were assessed with Agilent 2100 Bioanalyzer. RNA samples with RNA integrity number (RIN) $>7$ were used for multiplexed library preparation with Nugen Ovation RNA-Seq System V2. All samples were multiplexed into a single pool to avoid batch effects (Auer and Doerge, 2010), and sequencing was performed on Illumina NextSeq 4000 for $2 \times 75$ yielding at least 45 million reads per sample. De-multiplexing was performed with Illumina Bcl2fastq 2 v 2.17 program. Reads were aligned to the mouse mm10 reference genome using the STAR spliced read aligner (Dobin et al., 2013) with default parameters and fragment counts were derived using HTSseq program. Approximately $70 \%$ of the reads mapped uniquely to the mouse genome and were used for subsequent analyses. Differential gene expression analysis was performed with Bioconductor packages edgeR (Robinson et al., 2010) with false discovery rate (FDR) threshold $<0.05$ (http://www.bioconductor.org). Lowly expressed genes that had $\mathrm{CPM}>3$ in at least four samples were filtered out. In addition, we have applied FPKM $>1$ as an additional threshold to exclude low expression genes for the analyses of differentially expression gene (DEG) numbers and canonical pathways. This value was chosen based on previously published literature (Hebenstreit et al., 2011; Uhlen et al., 2017). For the top 50 gene list, we used FPKM $>5$ as a threshold to select genes with higher expression levels. Significantly associated canonical pathways $(p<0.05)$ were identified by ingenuity pathway analysis (IPA) using DEGs with thresholds FPKM $>1$ and FDR $<0.05$. The RNA-seq data have been deposited within the GEO repository (www.ncbi.nlm.nih.gov/geo) with accession number GSE164235. The complete list of DEGs is provided in Extended Data Table 7-1.

The cerebellar scRNA-seq data were downloaded from Linnarsson lab's database (www.mousebrain.org) and analysis was performed with the Scanpy package in Python (Wolf et al., 2018). Cerebellar cells with $>300$ genes and genes expressed in more than three cells were used for the subsequent analysis. Principal component analysis (PCA) was performed on the expression data matrix using Arpack wrapper function in the Scanpy package (Wolf et al., 2018). PCs 1-50 were used for generating $t$-distributed stochastic neighbor embedding (tSNE) plots. Eleven transcriptomic clusters were identified from total 11,063 cerebellar cells with a Louvain-Jaccard graph clustering algorithm with resolution set to
0.1 and were then annotated based on the expression of cell lineage marker genes (Saunders et al., 2018).

\section{Experimental design and statistical analyses}

For each experiment, multiple cohorts of age-matched mice with mixed genders were used to avoid batch effects. Comparisons were made as following: $\mathrm{CalEx}{ }^{\text {flox/wt }}$ mice with Cre AAV versus $\mathrm{CalEx}{ }^{\text {flox/wt }}$ mice with no AAV (Fig. 1); CalEx ${ }^{\text {flox/wt }}$ mice with Cre AAV versus $\mathrm{CalEx}^{\text {flox/wt }}$ mice with control AAV (Figs. 2, 6); bigenic mice with tamoxifen injection (multiple doses and single dose) versus littermate control mice including wild-type mice, Aldh1l1-Cre/ERT2 mice, and CalEx ${ }^{\text {flox/wt }}$ mice with tamoxifen injection (Figs. 3-5); bigenic mice with single dose tamoxifen injection versus littermate $\mathrm{CalEx}{ }^{\text {flox/wt }}$ mice with single dose tamoxifen injection (Fig. 7). The genotypes of mice were blind to the experimenters, however, bigenic mice developed observable behavioral phenotypes several days after tamoxifen injection, which made blinding the experiments difficult. Sample sizes were based on similar previously published work (Chai et al., 2017; Yu et al., 2018, 2020b; Nagai et al., 2019).

The results of statistical comparisons, $\mathrm{n}$ numbers and exact $p$ values are provided in the text, with the exception of $p<0.0001$. When the average data are reported in the text, the statistics are reported there. Statistical tests were performed in OriginPro 2016. Summary data are presented as mean \pm SEM along with the individual data points. Note that in some of the graphs the bars representing the SEM are smaller than the symbols used to represent the mean. For each set of data to be compared we determined whether the data were normally distributed or not with a Shapiro-Wilk test. If the data were normally distributed, we used parametric tests. If the data were not normally distributed, we used non-parametric tests. Two-tailed Student's $t$ tests with Welch correction and two-tailed Mann-Whitney tests were used for most statistical analyses. Comparisons of datasets with more than two groups or conditions were calculated with one-way ANOVA tests followed by post hoc Bonferroni tests. Survival curves were analyzed with Kaplan-Meier estimate. Significance was declared at $p<0.05$. In the figures, $p$ values were stated by asterisk(s) for simplification: $* p<0.05, * * p<0.01$, $* * * p<0.001$; n.s., not significant. $N$ is defined as the numbers of cells, sections or mice throughout on a case-by-case basis; the unit of analysis is stated in each figure panel, in the text or figure legend.

\section{Results}

There are two main sections to this study. In part one, we describe the generation of $\mathrm{CalEx}^{\text {flox }}$ mice, and their functional validation in the striatum (Figs. 1, 2). In part two, we used $\mathrm{CalEx}^{\text {flox }}$ mice to achieve CNS-wide attenuation of astrocyte $\mathrm{Ca}^{2+}$ signaling and explored the impact on mouse behavior and transcriptomic signatures of a relevant brain area that was suggested by our empirical observations, the cerebellum (Figs. 3-7). We also interpret the underlying changes following CalEx expression as a $\mathrm{Ca}^{2+}$ signaling attenuation tool using three descriptive models (Fig. 8). The RNA-seq data have been deposited within the GEO repository (www.ncbi.nlm.nih.gov/geo) with accession number GSE164235. The complete list of DEGs is provided in Extended Data Table 7-1.

\section{Generation and validation of knock-in $\mathrm{CalEx}^{\text {flox }}$ mice Generation of CalEx flox $^{\text {mice }}$}

$\mathrm{hPMCA} 2 \mathrm{w} / \mathrm{b}$ is an exogenous constitutively active pump that extrudes cytosolic $\mathrm{Ca}^{2+}$ (Fig. $1 A$ ) and results in attenuated astrocyte $\mathrm{Ca}^{2+}$ signaling (Yu et al., 2018). CalEx ${ }^{\text {flox }}$ knock-in mice harbor a CMV-IE enhancer/chicken $\beta$-actin/rabbit $\beta$-globin hybrid promoter (CAG), followed by a loxP-3xSV40pA-loxP STOP cassette and the mCherry-hPMCA2w/b cDNA at the Rosa26 locus (Madisen et al., 2015; Fig. 1A,B; Materials and Methods). Following four rounds of blastocyst injections with suitably validated recombinant JM8N4 ES cell clones, two founder lines of $\mathrm{CalEx}^{\text {flox }}$ knock-in mice were generated $\left(\mathrm{CalEx}^{\text {flox }}\right.$ 
A
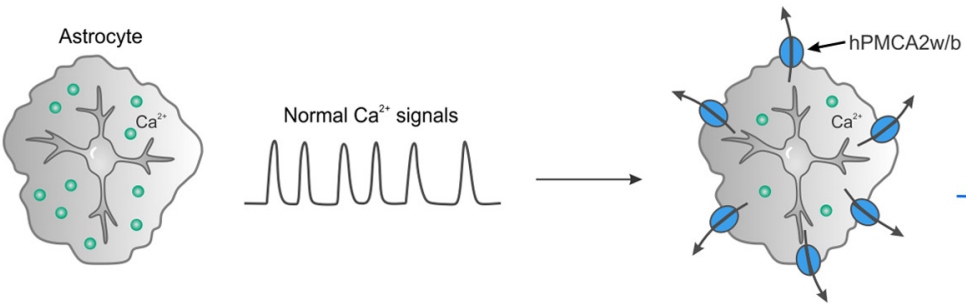

Reduced $\mathrm{Ca}^{2+}$ signals

B

Endogenous Rosa26 locus
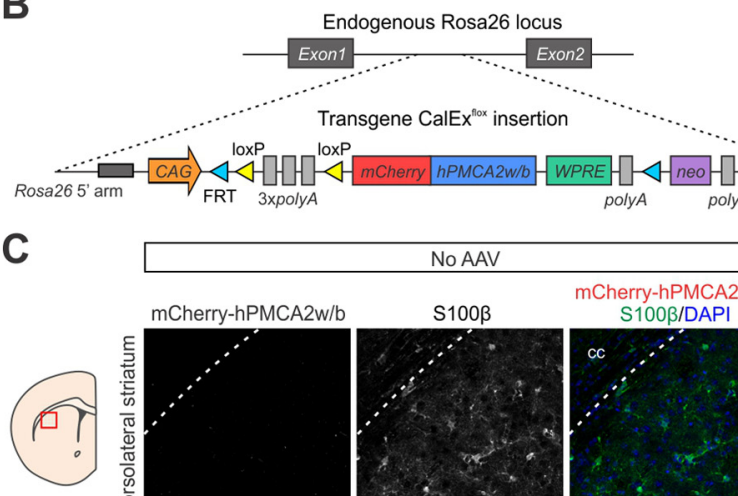
(1) CalEx ${ }^{\text {floxim }}$ mouse $A A V 2 / 5-G f a A B C, D-C r e$

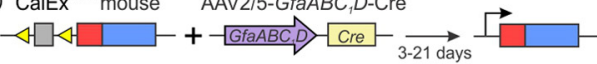
(2) CalEx ${ }^{\text {noxim }}$ mouse Aldh111-Cre/ERT2 mouse $\triangleleft \square \triangleleft \square-\times-\sqrt{A / d h 111}-\sqrt{\text { CreERT2 }}-\underset{\text { Tamoxifen }}{\longrightarrow}+\square$ Rosa26 5' arm
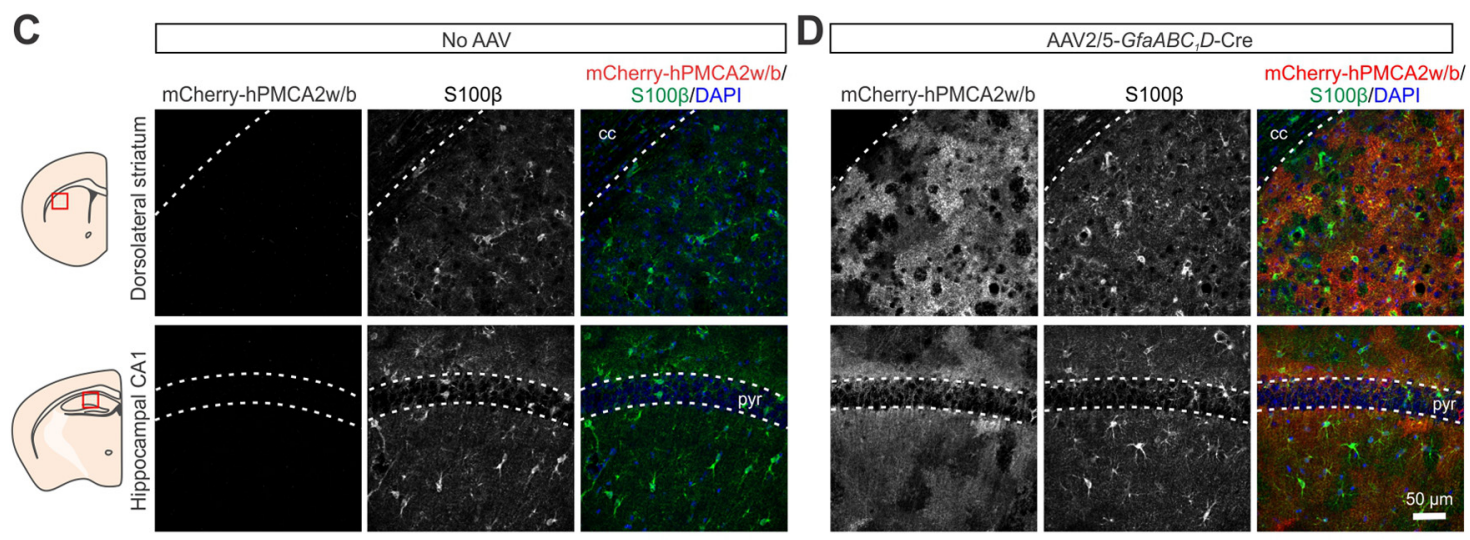

E

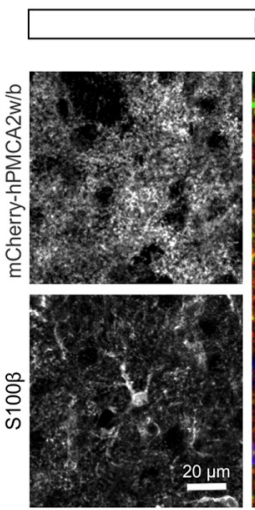

Dorsolateral striatum

$F$

CA2w/b/S100ß/DAP

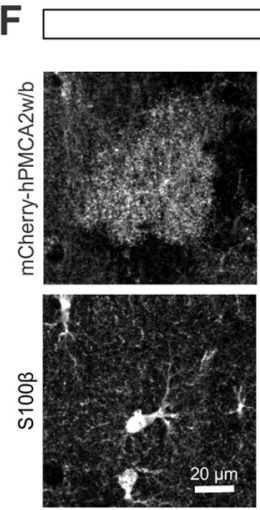

Hippocampal CA1

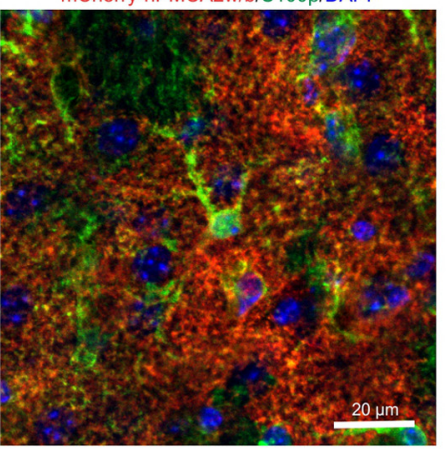

G

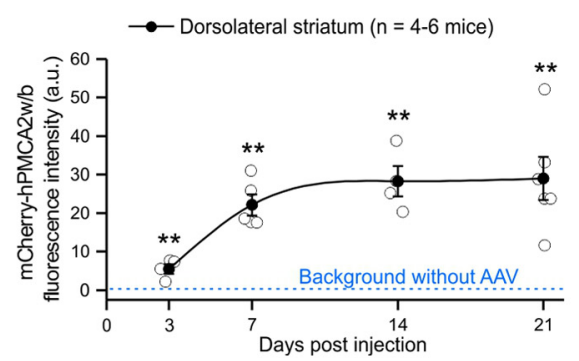

H

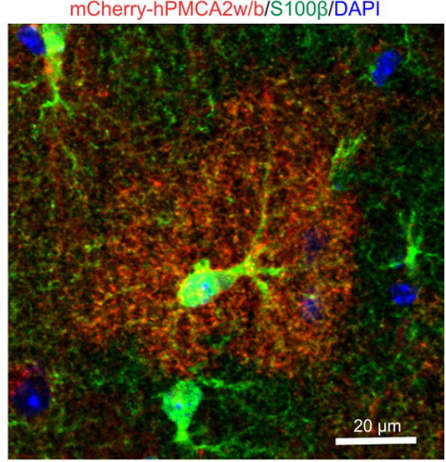

H $\rightarrow$ CA1 stratum radiatum $(n=4-6$ mice $)$

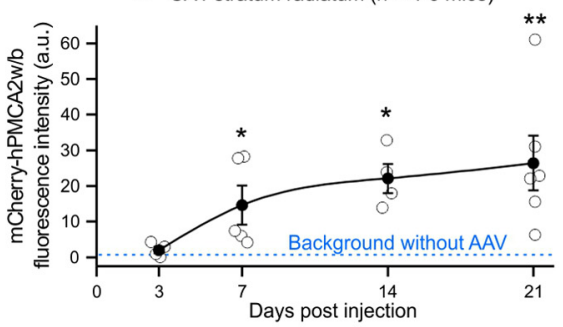

Figure 1. Generation of CalEx ${ }^{\text {flox }}$ mice to express $\mathrm{mCherry-hPMCA2w/b}$ in astrocytes for $\mathrm{Ca}^{2+}$ signaling attenuation. $\boldsymbol{A}$, Schematic for human plasma membrane $\mathrm{Ca}^{2+} \mathrm{ATPase}^{2}$ isoform 2 splice variant w/b (hPMCA2w/b) expression on the plasma membrane of astrocytes to attenuate $\mathrm{Ca}^{2+}$-dependent signaling. $\boldsymbol{B}$, Schematic of the generation and characterization of CalEx ${ }^{\text {flox }}$ mice. Left, CalEx ${ }^{\text {flox }}$ mice contain a CAG promoter, followed by a loxP-3×SV40pA-loxP cassette, and the mCherry-tagged hPMCA2w/b transgene (Yu et al., 2018) at the endogenous Rosa26 locus. Right, The expression of mCherry-hPMCA2w/b in astrocytes of adult mice was examined with two strategies: (1) an AAV2/5 encoding a Cre recombinase under an astrocyte-specific promoter $G f a A B C_{1} D$ was microinjected into a specific brain region; and (2) CalEx ${ }^{\text {flox }}$ mice were crossed with Aldh1/1-Cre/ERT2 mice to generate bigenic mice carrying both alleles (CalEx flox/wt; Aldh1/7-Cre/ERT2 mice). Upon the injection of tamoxifen, mCherry-hPMCA2w/b was expressed across the entire mouse brain. C, Representative IHC images from the dorsolateral striatum (top panels) and the hippocampal CA1 (bottom panels) of CalEx flox mice showing no leaky expression of mCherry-hPMCA2w/b in the absence of Cre recombinase. $D$, IHC images showing robust expression of $m$ Cherry-hPMCA2w/b in the dorsolateral striatum (top panels) and the hippocampal CA1 (bottom panels) of CalEx flox mice one week after the injection of AAV2/5-GfaABC ${ }_{1} D$-Cre. $\boldsymbol{E}, \boldsymbol{F}$, Higher-magnification IHC images showing co-localization of mCherry-hPMCA2w/b with astrocyte marker $5100 \beta$ in the dorsolateral striatum (E) and the hippocampal CA1 stratum radiatum $(\boldsymbol{F}) . \boldsymbol{G}, \boldsymbol{H}$, Timeline of mCherry-hPMCA2w/b expression in the dorsolateral striatum $(\boldsymbol{G})$ and the hippocampal CA1 stratum radiatum $(\boldsymbol{H})$ after AAV2/5-GfaABC ${ }_{1} D-C$ re injection. Data are shown as mean \pm SEM. In some cases, the SEM symbol is smaller than the symbol for the mean; $* p<0.05, * * p<0.01$. 
A
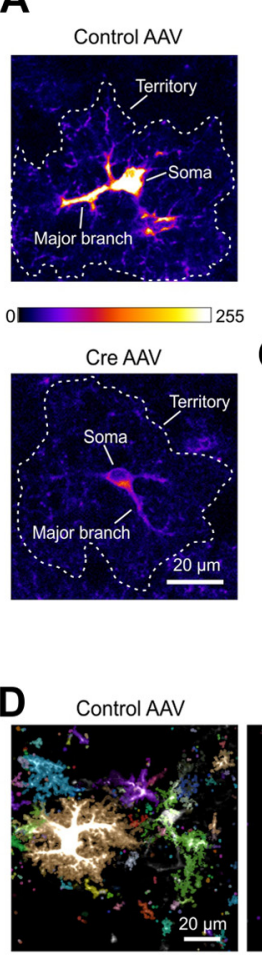

D

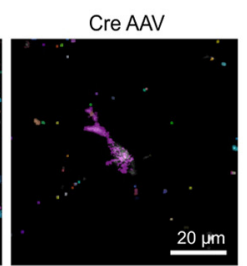

E

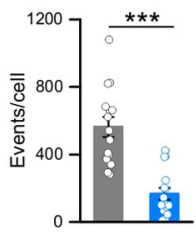

$\mathbf{F}$

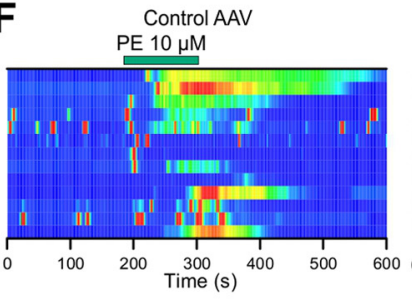

$\mathrm{H}$

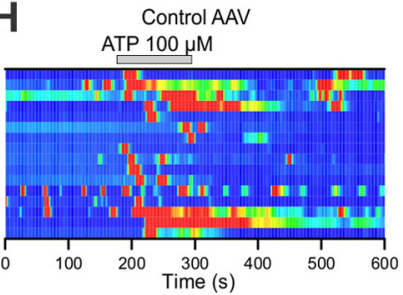

Control AAV

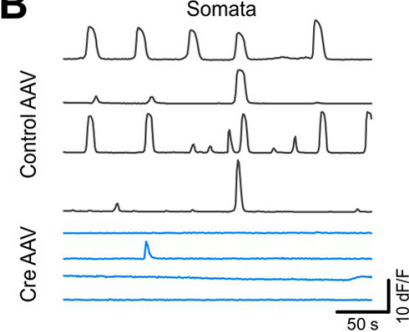

C

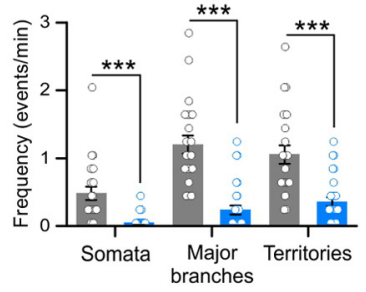

Control AAV ( $n=4$ mice, 22 cells)

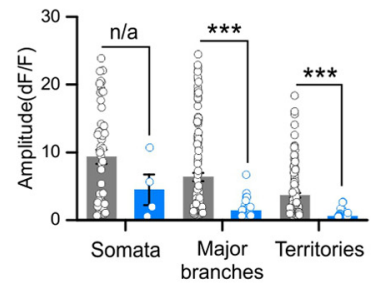

Control AAV ( $n=4$ mice, 31 cells)

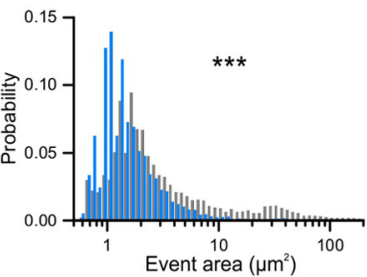

G

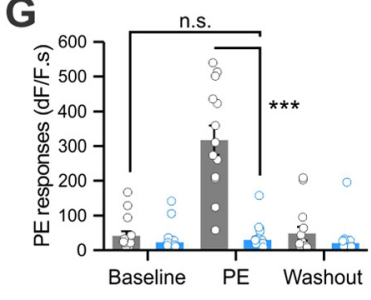

Control AAV

( $n=4$ mice, 13 cells)

Cre AAV

$(n=5$ mice, 17 cells $)$

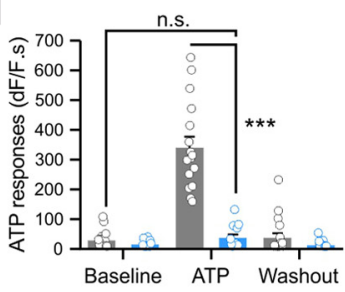

Control AAV

( $n=4$ mice, 16 cells)

Cre AAV

( $n=4$ mice, 11 cells $)$

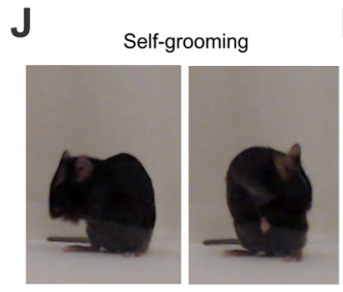

K

Self-grooming Non-grooming

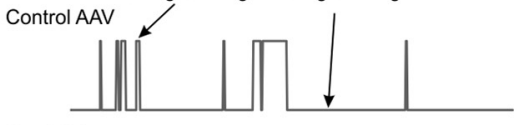

Cre AAV
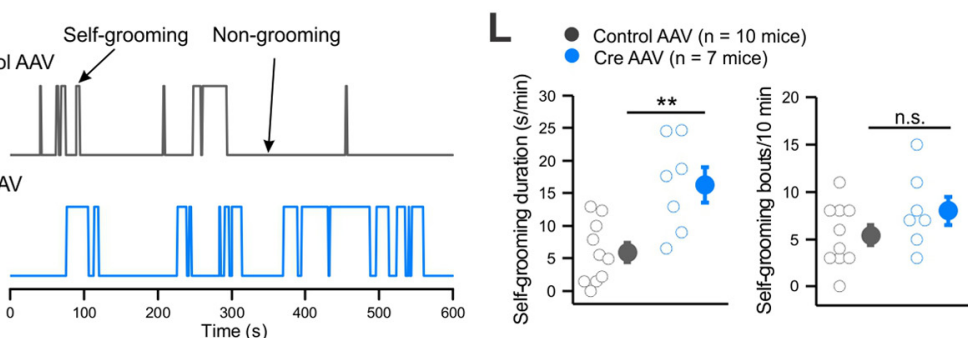

Figure 2. Attenuation of spontaneous and GPCR-mediated $\mathrm{Ca}^{2+}$ signaling in striatal astrocytes with CalEx ${ }^{\text {flox }}$ mice. $A$, Z-stack images of two-photon $\mathrm{Ca}^{2+}$ imaging of GCaMP6f-expressing striatal astrocytes from CalEx ${ }^{\text {flox }}$ mice microinjected with control (top) or Cre (bottom) AAVs, with the soma, major branch and territory outlined. $\boldsymbol{B}$, Representative traces of spontaneous Ca ${ }^{2+}$ signals in the somata, major branches and territories of striatal astrocytes from CalEx ${ }^{\text {flox }}$ mice with control (top) or Cre (bottom) AAVs. C, Frequency, peak amplitude and half-width of spontaneous $\mathrm{Ca}^{2+}$ signals in somata, major branches and territories of striatal astrocytes. D, Z-stack images of 10 -s $\mathrm{Ca}^{2+}$ recording from striatal astrocytes of CalEx ${ }^{\text {flox }}$ mice with control or Cre AAVs. Spontaneous $\mathrm{Ca}^{2+}$ events detected by AQuA were labeled in different colors. $\boldsymbol{E}$, Average numbers of $\mathrm{Ca}^{2+}$ events, distribution of event area sizes and spatial density of $\mathrm{co}_{0}-\mathrm{occurred} \mathrm{Ca}^{2+}$ events, detected by AQuA from 1-min recording. $\boldsymbol{F}$, Kymographs of $\mathrm{Ca}^{2+}$ responses evoked by PE $(10 \mu \mathrm{m})$ in the somata of striatal astrocytes from CalEx ${ }^{\text {flox }}$ mice with control or Cre AAVs. G, Quantification of PE-induced $\mathrm{Ca}^{2+}$ responses as integrated areas during baseline, PE application, and washout. $\boldsymbol{H}$, Kymographs of $\mathrm{Ca}^{2+}$ responses evoked by ATP (100 $\left.\mu \mathrm{M}\right)$ in the somata of striatal astrocytes from CalEx ${ }^{\text {flox }}$ mice with control or Cre AAVs. I, Quantification of ATP-induced $\mathrm{Ca}^{2+}$ responses as integrated areas during baseline, ATP application, and washout. J, Example images showing self-grooming behavior. $\boldsymbol{K}$, Behavioral traces of CalEx ${ }^{\text {flox }}$ mice with control or Cre AAVs showing self-grooming and non-grooming episodes over $10 \mathrm{~min}$. $L$, The duration of self-grooming (left) and the number of self-grooming bouts (right) of CalEx ${ }^{\text {flox }}$ mice with control or Cre AAVs. Average data are shown as mean $\pm \mathrm{SEM} ; * * p<0.01, * * * p<0.001$, n.s. $p>0.05$, n/a not applicable because of unequal sample sizes. 
A CalEx ${ }^{\text {noxmm }}$

(1) Tamoxifen (75 mg/kg for 5 days)

(2) Tamoxifen (75 mg/kg for 1 day)

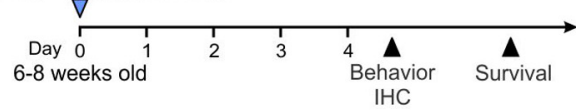

D

- Wild-type (6 mice)

Aldh1/1-Cre/ERT2 (4 mice)

- CalEx $x^{\text {loximt }}(6$ mice)

- CalEx ${ }^{\text {10xim, }} ;$ Aldh 1/1-Cre/ERT2 (6 mice)

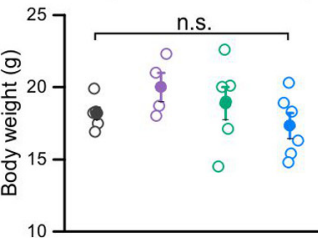

G

- Wild-type (8 mice)

- Aldh1/1-Cre/ERT2 (9 mice)

- CalEx $x^{\text {noximt }}$ (5 mice)

- CalEx ${ }^{\text {noxmim }} ;$ Aldh1/1-Cre/ERT2 (9 mice)

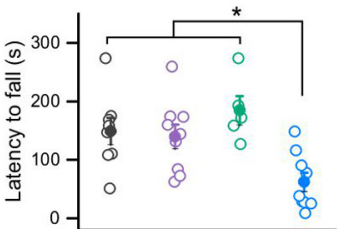

$\mathrm{J}$

Wild-type (8 mice)

Aldh1/1-Cre/ERT2 (10 mice)

- CalEx ${ }^{\text {floximt }}$ (11 mice)

- CalEx $x^{\mathrm{flox} / \mathrm{m} t} ;$ Aldh1/1-Cre/ERT2 (18 mice)

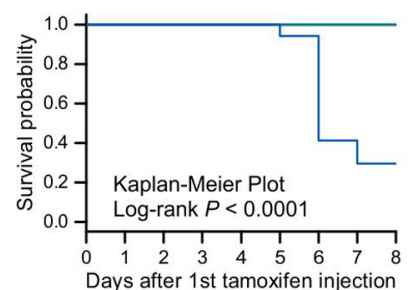

M Wild-type (8 mice)

Aldh1/1-Cre/ERT2 (8 mice)

- CalEx $x^{\text {floxwt }}$ (8 mice)

- CalEx ${ }^{\text {noximl; }} ;$ Aldh1/1-Cre/ERT2 (10 mice)

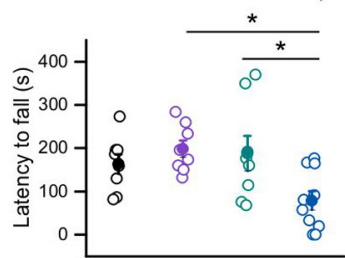

E Open-field test

Control CalEx ${ }^{\text {noxim: }} ;$ Aldh1/1-Cre/ERT2
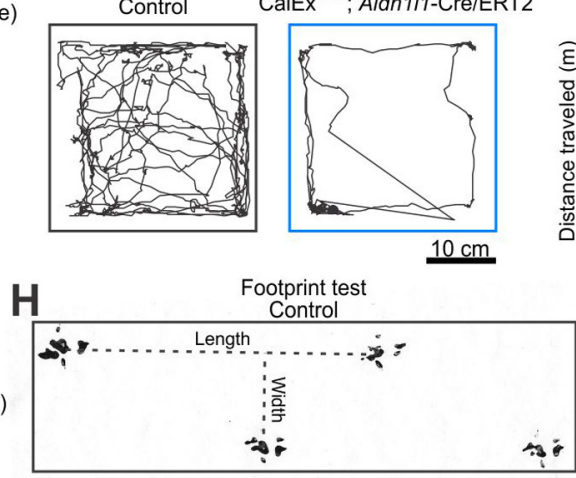

CalEx ${ }^{\text {flox/m}} ;$ Aldh1/1-Cre/ERT2

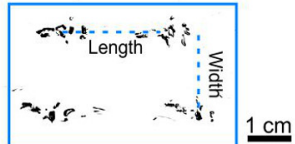

$\mathrm{K}$

Wild-type (4 mice)

Aldh1/1-Cre/ERT2 (5 mice)

- CalEx ${ }^{\mathrm{H} o x \mathrm{~m}}(7 \mathrm{mice})$

- CalEx ${ }^{\mathrm{Hox} / \mathrm{m}} ;$ Aldh1/1-Cre/ERT2 (7 mice)

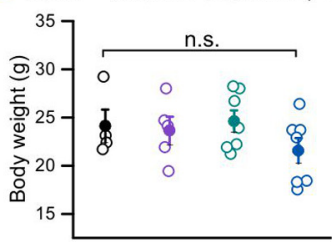

$\mathbf{N}$

- Wild-type (7 mice) Aldh1/1-Cre/ERT2 (5 mice)

- CalEx ${ }^{\text {loxim }}$ (6 mice) CalEx ${ }^{\text {mox/mt }} ;$ Aldh1/1-Cre/ERT2 (8 mice)

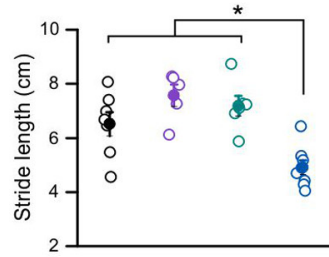

L

- Wild-type (7 mice)

CalEx $x^{\text {loximt }}(8$ mice)
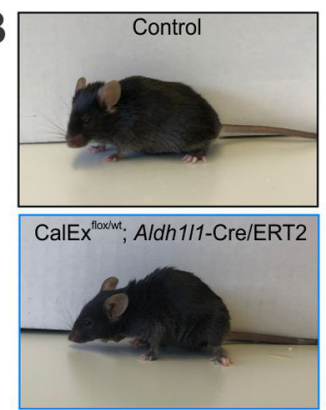

F Wild-type (6 mice) CalEx ${ }^{\text {foxwt }}$ (9 mice)

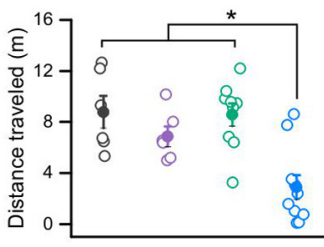

Wild-type (6 mice)

- Aldh1/1-Cre/ERT2 (6 mice)

CalEx ${ }^{\text {noxiw }}(9$ mice)

- CalEx $x^{\text {loxwt; }}$ Aldh1/1-Cre/ERT2 (9 mice)
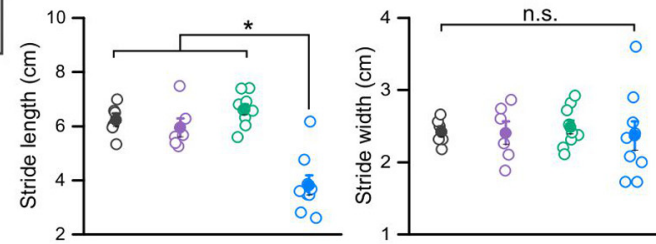

Open-field test

Aldh1/1-Cre/ERT2 (8 mice)
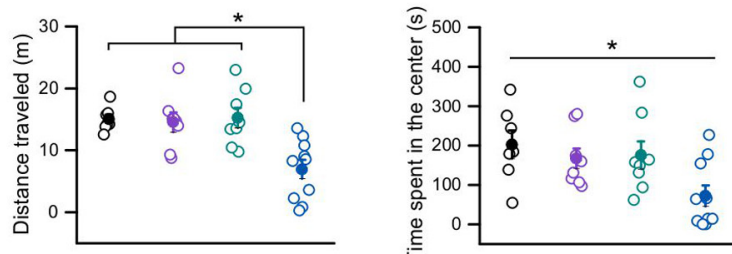

Figure 3. Expression of mCherry-hPMCA2w/b in adult CNS astrocytes in bigenic (CalEx ${ }^{\text {flox; }}$ Aldh117-Cre/ERT2) mice results in strong behavioral phenotypes and premature lethality. A, Schematic of experimental design. CalEx flox mice were crossed with Aldh1/1-Cre/ERT2 mice to generate bigenic (CalEx flox/wt; Aldh117-Cre/ERT2) mice, which received tamoxifen intraperitoneally at $75 \mathrm{mg} / \mathrm{kg}$ body weight for $1 \mathrm{~d}$ or five consecutive days. The first dose of tamoxifen was administered on day 0 , behavioral and IHC assessments were performed on day 4 . Behavioral results for multiple doses of tamoxifen injection $(75 \mathrm{mg} / \mathrm{kg} \times 5 \mathrm{~d}$ ) are summarized in panels $\boldsymbol{B}-\boldsymbol{I}$ and results for single dose tamoxifen injection $(75 \mathrm{mg} / \mathrm{kg} \times 1 \mathrm{~d})$ are summarized in panels $\mathbf{J}-\mathbf{N}$. $\boldsymbol{B}$, Images of a control mouse and a bigenic mouse after the last dose of tamoxifen injection. C, Survival probability of littermate control (wild-type, CalEx ${ }^{\text {flox/wt }}$ and Aldh117-Cre/ERT2) and bigenic mice with multiple doses of tamoxifen. $\boldsymbol{D}$, Body weight of control and bigenic mice was comparable. $\boldsymbol{E}$, Representative track traces of locomotor activity in open-field tests of control and bigenic mice over $10 \mathrm{~min}$. $\boldsymbol{F}$, Travel distance (left) and time spent in the center (right) of open-field activity. Bigenic mice traveled significantly less distance compared with control mice. $\boldsymbol{G}$, Average latency to fall in a rotarod test of control and bigenic mice. $\boldsymbol{H}$, Representative footprint tracks of control and bigenic mice. Dotted lines indicate stride lengths and widths. $\boldsymbol{I}$, Average footprint stride length and width of control and bigenic mice. J, Survival probability of littermate control and bigenic mice with single dose tamoxifen. $\boldsymbol{K}$, Body weight of control and bigenic mice was similar. $\boldsymbol{L}$, Bigenic mice traveled significantly less distance (left) and spent less time in the center of the open-filed chamber (right) compared with control mice. $\boldsymbol{M}$, Average latency 
mice; lines 1 and 2). The insertion of the mCherry-hPMCA2w/b transgene was confirmed by PCR of genomic DNA from tail biopsies (Materials and Methods). Both lines underwent germline transmission, with line 1 producing slightly higher expression of mCherry-hPMCA2w/b protein than line 2 from initial characterization with IHC. The founders from line 1 were thus backcrossed with C57BL/6NTac mice for more than eight generations for subsequent experimental validations. Both homozygous and heterozygous $\mathrm{CalEx}^{\text {flox }}$ mice were viable and fertile without any noticeable behavioral alterations. In this study, we characterized heterozygous $\mathrm{CalEx}^{\text {flox }}$ mice from line 1 in detail with either Cre AAV microinjections or with crosses to Aldh1l1Cre/ERT2 expressing mice. Line $1 \mathrm{CalEx}^{\text {flox }}$ mice have been deposited at JAX with descriptor Gt(ROSA)26Sor tm1(CAG-mCherryhPMCA2w/b)Khakh (Stock No. 035252).

Immunohistochemical (IHC) validation of CalEx flox mice. To validate $\mathrm{CalEx}^{\text {flox }}$ mice for attenuating astrocyte $\mathrm{Ca}^{2+}$ signaling, we generated brain region-specific expression using an AAV2/5 encoding Cre recombinase under the astrocyte promoter $G f a A B C_{1} D$. With this AAV strategy, experiments were performed 3-21 d after microinjections into $\sim 56 \mathrm{~d}$ old adult mice (Fig. 1B). Based on past work and our research interests, we chose the dorsal striatum and the hippocampal CA1 region to evaluate CalEx ${ }^{\text {flox }}$ mice (Srinivasan et al., 2016; Chai et al., 2017). We detected no mCherry-hPMCA2w/b expression in $\mathrm{CalEx}^{\text {flox }}$ mice in the absence of Cre ( $n=5$ and 6 mice for the striatum and the hippocampal CA1, respectively; Fig. $1 C$ ). In contrast, microinjection of AAV2/5- $G f a A B C_{1} D$-Cre induced robust mCherryhPMCA $2 \mathrm{w} / \mathrm{b}$ expression in both the dorsolateral striatum and the hippocampal CA1 regions of adult $\mathrm{CalEx}^{\text {flox }}$ mice $(n=5$ mice for both regions; Fig. 1D). The mCherry-hPMCA2w/b expressing cells resembled astrocytes in their morphology and the expression of the transgene co-localized with $\mathrm{S} 100 \beta$, which labels astrocytes in these brain regions (Srinivasan et al., 2016; Chai et al., 2017). Notably, the bushy nature of mCherryhPMCA2w/b expressing cells (Fig. $1 E, F$ ) was similar to that of astrocytes expressing plasma membrane targeted fluorescent proteins (Benediktsson et al., 2005; Shigetomi et al., 2013; Srinivasan et al., 2016).

We next examined the time course of mCherry-hPMCA2w/b expression 3, 7, 14, and $21 \mathrm{~d}$ after microinjection of Cre AAVs into the striatum or hippocampus (Fig. $1 G, H$ ). We observed significant mCherry-hPMCA2w/b expression $7 \mathrm{~d}$ after AAV microinjections in both brain regions $(n=5$ mice per region, for the striatum: $U=0, p=0.005$ compared with background intensity; for the hippocampus: $U=1, p=0.02$ compared with background intensity; Mann-Whitney test). Subsequently, expression levels increased slightly and were maintained at $14 \mathrm{~d}(n=4$ mice per region, for the striatum: $U=0, p=0.009$ compared with background intensity; for the hippocampus: $U=0, p=0.018$ compared with background intensity; Mann-Whitney test) and $21 \mathrm{~d}$ ( $n=6$ mice per region, for the striatum: $U=0, p=0.004$ compared with background intensity; for the hippocampus: $U=0$, $p=0.008$ compared with background intensity; Mann-Whitney test; Fig. $1 G, H$ ). Expression in the striatum (but not the hippocampus) was significant $3 \mathrm{~d}$ after Cre AAV microinjections

to fall in a rotarod test of control and bigenic mice. $\boldsymbol{N}$, Average footprint stride length and width of control and bigenic mice. Average data are shown as mean \pm SEM; $* p<0.05$, n.s. $p>0.05$. The $p$ values were from one-way ANOVA with a post hoc Bonferroni test.

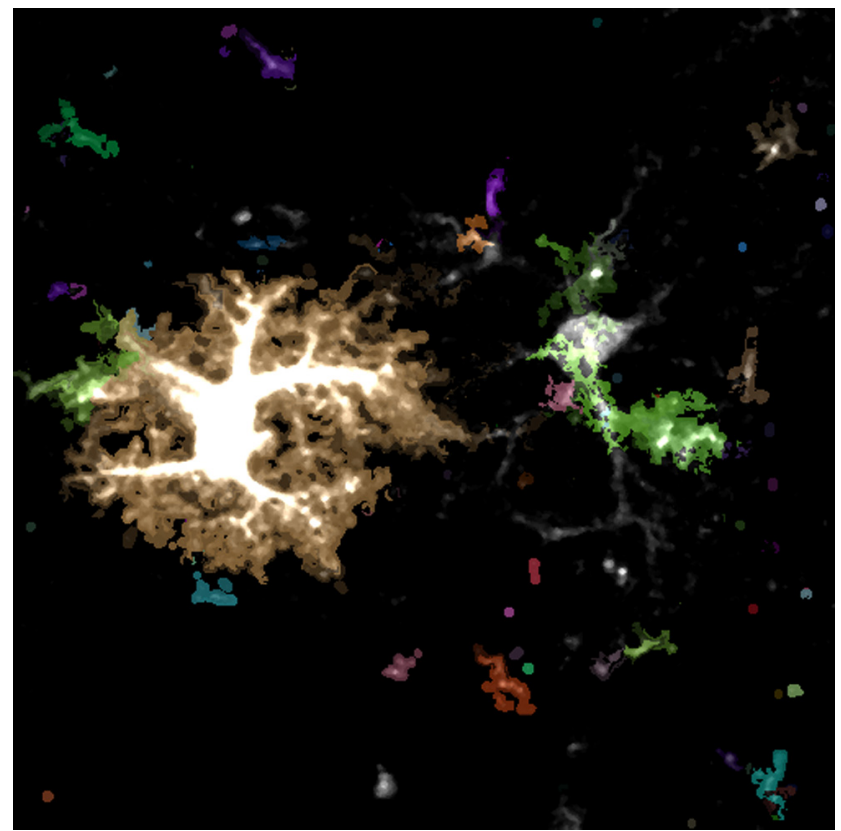

Movie 1. A 1-min recording of spontaneous $\mathrm{Ca}^{2+}$ signals in control striatal astrocytes from a CalEx ${ }^{\text {flox }}$ mouse with events colored by AQuA. [View online]

( $n=4$ mice per region, for the striatum: $U=0, p=0.009$ compared with background intensity; for the hippocampus: $U=8.5$, $p=0.80$ compared with background intensity; Mann-Whitney test; Fig. $1 G)$. Thus, CalEx ${ }^{\text {flox }}$ mice result in faster expression and improve on limitations with $G f a A B C_{1} D$-mCherry-hPMCA2w/b AAVs (Yu et al., 2018), which typically require two to three weeks of expression. The IHC experiments show that CalEx ${ }^{\text {flox }}$ mice can be used to express mCherry-hPMCA2w/b in a cell type-specific and brain region-specific manner.

Functional validation of CalEx flox mice with $\mathrm{GfaABC}_{1} \mathrm{D}-\mathrm{Cre}$ $A A V 2 / 5$. For the functional validation experiments with CalEx ${ }^{\text {flox }}$ mice, we focused on the striatum because of the availability of past data for comparisons (Jiang et al., 2016; Yu et al., $2018,2020 \mathrm{~b})$. We evaluated whether expression of mCherryhPMCA2w/b from CalEx ${ }^{\text {flox }}$ mice affected striatal astrocyte $\mathrm{Ca}^{2+}$ signaling and animal behavior and compared the data to published work (Yu et al., 2018, 2020b). Delivery of two $G f a A B C_{1} D$ promoter AAVs results in high co-expression of the cognate cargo from each AAV in the same population of striatal astrocytes (Srinivasan et al., 2016; Chai et al., 2017; Octeau et al., 2018; Yu et al., 2018; Diaz-Castro et al., 2019; Nagai et al., 2019). Thus, we microinjected AAV2/5-GfaABC $C_{1} D$-GCaMP6f to express the fast $\mathrm{Ca}^{2+}$ indicator GCaMP6f (Chen et al., 2013) together with either a control AAV (AAV2/5-G $f a A B C_{1} D$-tdTomato) or a Cre AAV (AAV2/5-G $f a A B C_{1} D$-Cre) into the dorsal striatum of adult CalEx ${ }^{\text {flox }}$ mice and performed $\mathrm{Ca}^{2+}$ imaging of striatal astrocytes in striatal slices with two-photon laser-scanning microscopy (Fig. 2A). Extensive spontaneous $\mathrm{Ca}^{2+}$ signals were detected and analyzed from the somata, major branches, and areas encompassing entire territories of striatal astrocytes (Fig. 2B). mCherry$\mathrm{hPMCA} 2 \mathrm{w} / \mathrm{b}$ expression significantly reduced the frequency of spontaneous $\mathrm{Ca}^{2+}$ signals in all compartments, which was clear from the representative traces (Fig. $2 B$ ) and from the individual and average data (Fig. $2 C$ ). The effect on the somata was most pronounced resulting in a decrease from $0.48 \pm 0.10$ events $/ \mathrm{min}$ with control AAV to $0.04 \pm 0.02$ events/min with Cre AAV $(n=22-27$ cells from $4-5$ mice, $U=521, p<0.0001$, Mann- 


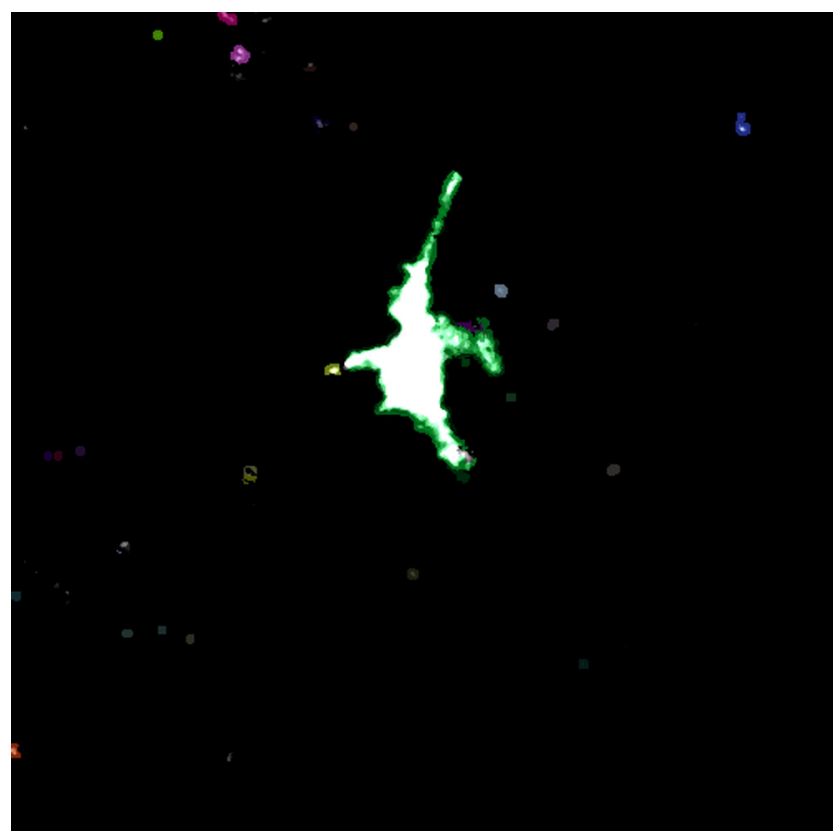

Movie 2. A 1-min recording of spontaneous $\mathrm{Ca}^{2+}$ signals in a Cre-expressing striatal astrocyte from a CalEx ${ }^{\text {flox }}$ mouse with events colored by AQuA. [View online]
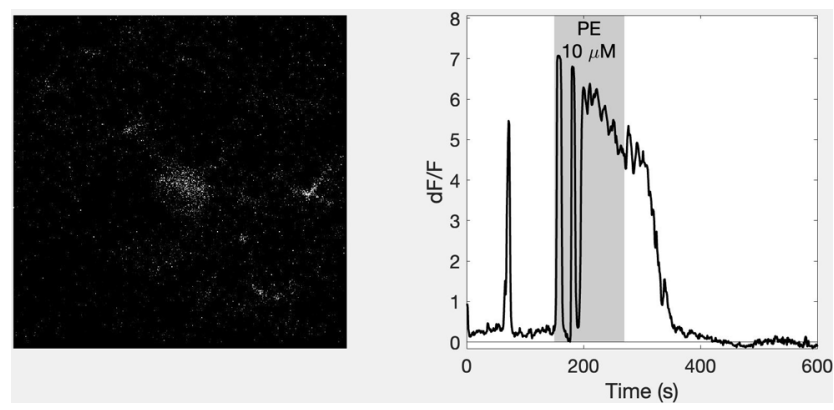

Movie 3. A 10-min recording of $\mathrm{Ca}^{2+}$ responses to $10 \mu \mathrm{M}$ PE in a control striatal astrocyte from a CalEx ${ }^{\text {flox }}$ mouse. [View online]

Whitney test; Fig. 2B,C). The frequency of spontaneous $\mathrm{Ca}^{2+}$ signals in the CalEx ${ }^{\text {flox }}$ mice following Cre AAV microinjections was also reduced in the major branches $(1.20 \pm 0.13$ events $/ \mathrm{min}$ with control AAV vs $0.23 \pm 0.07$ events/min with Cre AAV, $U=552.5, p<0.0001$, Mann-Whitney test) and territories of striatal astrocytes $(1.05 \pm 0.13$ events/min with control AAV vs $0.36 \pm 0.07$ events $/ \mathrm{min}$ with Cre AAV, $U=506.5, p<0.0001$, Mann-Whitney test). Moreover, the amplitudes and durations of spontaneous $\mathrm{Ca}^{2+}$ signals were all attenuated in striatal astrocytes in $\mathrm{CalEx}^{\text {flox }}$ mice following AAV Cre microinjections (Fig. $2 B, C)$.

To capture the spatiotemporal dynamics of complex $\mathrm{Ca}^{2+}$ signals, we used recently developed software called astrocyte quantitative analysis (AQuA; Wang et al., 2019), and performed event-based analysis (Fig. 2D). Over $60 \mathrm{~s}$ of imaging, we detected significantly fewer events in striatal astrocytes from $\mathrm{CalEx}^{\text {flox }}$ mice with Cre AAV compared with those with control AAV $(168 \pm 35$ events/cell vs $564 \pm 59$ events/cell, $n=29-31$ cells from 4-5 mice, $U=211, p<0.0001$, Mann-Whitney test; Fig. $2 E$; Movies 1, 2). In striatal astrocytes expressing CalEx, the areas occupied by the largest $\mathrm{Ca}^{2+}$ events, which represent waves, were significantly reduced ( $p<0.0001$, Kruskal-Wallis test; Fig.
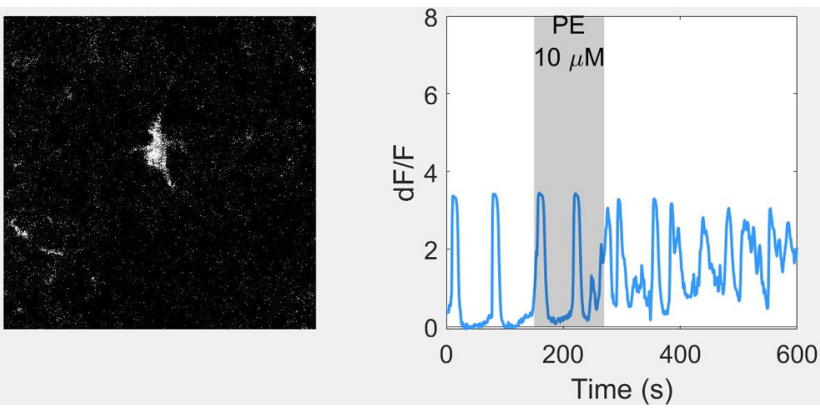

Movie 4. A 10-min recording of $\mathrm{Ca}^{2+}$ responses to $10 \mu \mathrm{M}$ PE in a Cre-expressing striatal astrocyte from a CalEx ${ }^{\text {flox }}$ mouse. [View online]

$2 E$ ). For instance, $\sim 10 \%$ of the total $\mathrm{Ca}^{2+}$ events measured in control astrocytes displayed areas larger than $20 \mu \mathrm{m}^{2}$, but following Cre-mediated mCherry-hPMCA $2 \mathrm{w} / \mathrm{b}$ expression this was $\sim 0.7 \%$. Consistent with the analysis from different cellular compartments (Fig. $2 A-C$ ), the probability of $\mathrm{Ca}^{2+}$ events displaying shorter durations was significantly higher in CalEx-expressing astrocytes $(p<0.0001$, Kruskal-Wallis test). Additionally, the network spatial density within $10 \mathrm{~s}$ time windows from the imaged area $(\sim 100 \times 100 \mu \mathrm{m})$ was significantly lower in CalEx expressing astrocytes $(p<0.0001$, Kruskal-Wallis test; Fig. $2 E)$. Together, these data show profound global reduction of $\mathrm{Ca}^{2+}$ signaling following mCherry-hPMCA2 $\mathrm{w} / \mathrm{b}$ expression in CalEx ${ }^{\text {flox }}$ mice irrespectively of whether the data are analyzed using ROIs (Fig. $2 A-C$ ) or event-based analysis methods (Fig. $2 D, E)$.

Astrocytes respond to the activation of diverse GPCRs by elevating intracellular $\mathrm{Ca}^{2+}$ levels (Porter and McCarthy, 1997; Fiacco et al., 2009). We next examined whether $\mathrm{CalEx}^{\text {flox }}$ mice can be used to attenuate GPCR-mediated responses in astrocytes in striatal slices. AAV Cre mediated mCherry-hPMCA2w/b expression in CalEx ${ }^{\text {flox }}$ mice resulted in significantly attenuated $10 \mu \mathrm{M}$ PE-evoked $\mathrm{Ca}^{2+}$ responses in astrocyte somata, major branches, and territories by $\sim 90 \%$ (Movies 3, 4; $n=13-17$ cells from 4-5 mice, for the somata: $U=217, p<0.0001$; for the major branches: $U=217, p<0.0001$; for the territories: $U=206$, $p<0.0001$, Mann-Whitney test; Fig. 2F,G). Similarly, $100 \mu \mathrm{M}$ ATP evoked astrocyte $\mathrm{Ca}^{2+}$ responses in striatal astrocytes were also reduced significantly by $\sim 90 \%$ ( $n=11-16$ cells from four mice, for the somata: $U=176, p<0.0001$, Mann-Whitney test; for the major branches: $U=176, p<0.0001$, Mann-Whitney test; for the territories: $t_{(15.31)}=6.58, p<0.0001$, Student's $t$ test with Welch correction; Fig. $2 H, I)$. In these settings, PE and ATP activate $\alpha 1$ adrenoceptors and a mixture of metabotropic ATP $\mathrm{P} 2 \mathrm{Y}$ receptors on striatal astrocytes, respectively (Chai et al., 2017; Yu et al., 2018; Nagai et al., 2019).

We previously demonstrated that attenuating spontaneous and GPCR-mediated $\mathrm{Ca}^{2+}$ signaling in striatal astrocytes of adult mice led to excessive self-grooming behaviors (Yu et al., 2018). To investigate whether mCherry-hPMCA2w/b expression from $\mathrm{CalEx}^{\text {flox }}$ mice can reproduce those findings, we microinjected Cre AAV bilaterally into the dorsal striatum and evaluated innate self-grooming behavior of the mice (Fig. $2 J-L$ ). CalEx ${ }^{\text {flox }}$ mice that received Cre AAV showed significantly longer self-grooming bouts compared with $\mathrm{CalEx}^{\text {flox }}$ mice that received control AAV $(16.3 \pm 2.7 \mathrm{~s}$ per min with Cre AAV vs $5.9 \pm 4.7 \mathrm{~s}$ per min with control AAV, $n=10$ and 7 mice, respectively, $t_{(15)}=-3.63$, $p=0.002$, Student's $t$ test; Fig. $2 K, L)$. As expected, the number of self-grooming bouts was not altered $\left(t_{(15)}=-1.47, p=0.16\right.$, 
Student's $t$ test; Fig. $2 L$ ). Overall, the effects of striatal astrocyte mCherry-hPMCA2w/b expression in CalEx ${ }^{\text {flox }}$ mice reproduced cellular and behavioral effects that were expected to result from attenuated astrocyte $\mathrm{Ca}^{2+}$ signaling in this brain region.

\section{Bigenic CalEx ${ }^{\mathrm{flox} / \mathrm{wt}} /$ Aldh1l1-Cre/ERT2 mice for CNS-wide attenuation of $\mathrm{Ca}^{2+}$ signals}

In light of the emerging data on region-specific astrocytes (Haim and Rowitch, 2017; Khakh and Deneen, 2019; Pestana et al., 2020), functions within neural circuits are best explored by performing circuit-specific astrocyte manipulations. This could be achieved with local striatal microinjections (Figs. 1, 2) or with genetic approaches that target regionally specific subpopulations of astrocytes (Huang et al., 2020), which do not yet exist for the striatum. Such local approaches are preferable to whole brain manipulations, where the consequences may be problematic to interpret (Khakh and McCarthy, 2015; Khakh, 2019; Yu et al., 2020a). However, a previous approach to abrogate astrocyte $\mathrm{Ca}^{2+}$ signaling with $\mathrm{IP}_{3} \mathrm{R} 2-/-$ mice in the whole CNS resulted in no changes in mouse behavior in the early explorations (Agulhon et al., 2008, 2010; Petravicz et al., 2008, 2014). This was interpreted to indicate that astrocyte $\mathrm{Ca}^{2+}$ signaling may not play a prominent physiological role within the brain. Subsequently, the discovery of additional types and residual $\mathrm{Ca}^{2+}$ signals in $\mathrm{IP}_{3} \mathrm{R} 2-/-$ mice motivated reconsideration of the original conclusions (Otsu et al., 2015; Srinivasan et al., 2015; Rungta et al., 2016; Agarwal et al., 2017; Bindocci et al., 2017), and several studies have used the same mice and detected changes in mouse behavior during more specialized investigations (Savtchouk and Volterra, 2018; Yu et al., 2020a; Nagai et al., 2021). As additional validation of CalEx ${ }^{\text {flox }}$ mice, we therefore explored the consequences of CNS-wide astrocyte $\mathrm{Ca}^{2+}$ attenuation by crossing CalEx ${ }^{\text {flox }}$ mice with Aldh1l1-Cre/ERT2 mice to generate bigenic mice in which tamoxifen triggered global mCherryhPMCA2w/b expression in adults (Srinivasan et al., 2016).

\section{Behavioral abnormalities in bigenic CalEx flox/wt/Aldh1l1-Cre/ ERT2 mice}

Since the expression of transgenes with Aldh1l1-Cre/ERT2 mice by tamoxifen was shown to be dose dependent (Srinivasan et al., 2016), we administrated tamoxifen using two regimens (multiple doses at $75 \mathrm{mg} / \mathrm{kg}$ body weight for five consecutive days, or a single dose for $1 \mathrm{~d}$; Fig. $3 A$ ). Four groups of adult mice at six to eight weeks old were used in the experiments: (1) wild-type mice; (2) Aldh1l1-Cre/ERT2 mice; (3) CalEx ${ }^{\text {flox/wt }}$ mice; and (4) bigenic (CalEx ${ }^{\text {flox/wt }}$; Aldh1l1-Cre/ERT2) mice. In the multiple dose tamoxifen group, $3 \mathrm{~d}$ after the initial dose of tamoxifen was administered, the bigenic mice deteriorated rapidly, displaying reduced mobility, ruffled fur, and hunched postures. However, their littermate controls were normal $(n=7-19$ mice; Fig. $3 B)$. We tracked several cohorts of mice, each comprising mixed numbers and genders of control and bigenic mice after tamoxifen administration, and found that survival decreased sharply in bigenic mice $6 \mathrm{~d}$ after the initial dose of tamoxifen $(n=7-19$ mice per group, $\chi^{2}=41.74, p<0.0001$, Kaplan-Meier estimate; Fig. $3 C$ ). Of the bigenic mice examined, $92 \%$ were dead by day 7 , whereas $97 \%$ of the controls were alive. Although the bigenic mice showed a trend of body weight loss close to the mortality end point, there were no statistical differences in body weight between groups $\left(n=4-6\right.$ mice per group, $F_{(3,18)}=1.41, p=0.27$, one-way ANOVA; Fig. 3D). In addition, we assessed behaviors related to exploration, motor coordination, and motor function.
In an open-field test, the bigenic mice displayed lower ambulation, traveling significantly less distance compared with control mice ( $n=6-10$ mice per group, $F_{(3,27)}=8.78, p=0.0003$, one-way ANOVA with a post hoc Bonferroni test; Fig. $3 E, F)$. The time spent in the center of the open-field arena was indiscernible among groups $\left(F_{(3,27)}=1.81, p=0.17\right.$, one-way ANOVA; Fig. $3 F)$. In a rotarod test, the latency for the bigenic mice to fall off the accelerating rod was significantly shorter than control mice, indicating altered motor coordination and balance $(n=5-9$ mice per group, $F_{(3,27)}=5.92, p=0.003$, one-way ANOVA with a post hoc Bonferroni test; Fig. $3 G$ ). To assess the gait of mice, we performed a hindlimb footprint test and found that bigenic mice showed significantly shorter stride lengths $(n=6-9$ mice per group, $F_{(3,26)}=20.62, p<0.0001$, one-way ANOVA with a post hoc Bonferroni test), but normal stride widths compared with control mice $\left(F_{(3,26)}=0.12, p=0.94\right.$, one-way ANOVA; Fig. $\left.3 H, I\right)$.

In the single dose tamoxifen group, the bigenic mice exhibited similarly declining/worsening appearances and high mortality $6 \mathrm{~d}$ after receiving the single dose of tamoxifen. Compared with the multiple dose group, the bigenic mice with low dose tamoxifen had extended survival, e.g., $29 \%$ of bigenic mice were alive on day 7 compared with $8 \%$ in multiple dose group (Fig. $3 J$ ). None of the control mice died during the course of experiments and body weight was similar among different groups $(n=4-7$ mice per group, $F_{(3,19)}=1.12, p=0.37$, one-way ANOVA; Fig. $\left.3 K\right)$. Similarly, bigenic mice traveled less distance in the open-field test because of decreased mobility ( $n=7-10$ mice per group, $F_{(3,29)}=8.28, p=0.0004$, one-way ANOVA with a post hoc Bonferroni test; Fig. $3 L$ ). Although the time spent in the center of the arena was significantly less in bigenic mice when comparing with wild-type littermates $\left(F_{(3,29)}=3.77, p=0.032\right.$ vs wildtype, one-way ANOVA with a post hoc Bonferroni test), it was not different from the other control groups ( $p=0.18$ vs Aldh1l1Cre/ERT2, $p=0.12$ vs CalEx ${ }^{\text {flox/wt }}$; Fig. $3 L$ ). Moreover, bigenic mice showed deteriorated performance in the rotarod test $\left(n=8-10\right.$ mice per group, $F_{(3,30)}=4.43, p=0.011$, one-way ANOVA with a post hoc Bonferroni test; Fig. $3 M$ ) and altered hindlimb gait ( $n=5-8$ mice per group, for stride length: $F_{(3,22)}=$ $10.50, p=0.0002$, for stride width: $F_{(3,22)}=0.94, p=0.44$, oneway ANOVA with a post hoc Bonferroni test; Fig. $3 N$ ). Together, our data suggest that astrocytic expression of CalEx in the CNS markedly affected physiology (see Discussion).

Preferentially strong cerebellar expression of mCherry$h P M C A 2 w / b$ in bigenic mice. To investigate the brain regions that may underlie behavioral alterations in bigenic mice, we surveyed the expression of mCherry-hPMCA2w/b across the CNS of bigenic mice treated either with multiple doses of tamoxifen or with a single dose. We found that mCherry-hPMCA2w/b was expressed throughout the brain and spinal cord of bigenic mice, but undetectable in all three control groups (Fig. 4A). Notably, the expression of mCherry-hPMCA2w/b within the cerebellum was particularly high and localized to the molecular layer where the Bergmann glia processes enwrap the synapses of the Purkinje neurons ( $n=3-5$ mice; Fig. $4 A)$. We further quantified mCherry-hPMCA2w/b expression from 14 CNS regions including the olfactory bulb $(\mathrm{Ob})$, the motor cortex $(\mathrm{MCx})$, the sensory cortex (SCx), the visual cortex (VCx), dorsal striatum (dSt), nucleus accumbens (NAc), hippocampal CA1, thalamus (Th), hypothalamus (Hy), substantia nigra ( $\mathrm{SNr}$ ), facial motor nucleus $(7 \mathrm{~N})$ in the hindbrain, cerebellum $(\mathrm{Cb})$, cervical spinal cord (CSc), and lumbar spinal cord (LSc; Fig. 4B,C). Across $14 \mathrm{CNS}$ regions, the percentage of $\mathrm{S} 100 \beta+$ cells expressing mCherryhPMCA2w/b ranged from $\sim 33 \%(\mathrm{MCx})$ to $94 \%(\mathrm{Cb}, n=3-5$ 


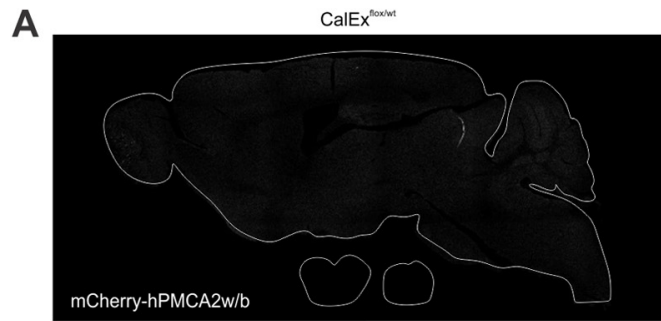

CalEx ${ }^{\text {hoxwirt; }}$ Aldh1/1-Cre/ERT2 (Tamoxifen $75 \mathrm{mg} / \mathrm{kg} \times 5$ )

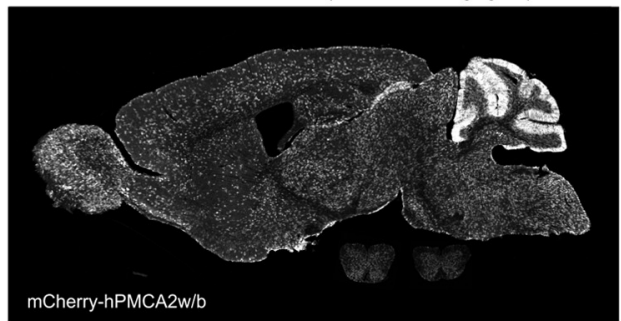

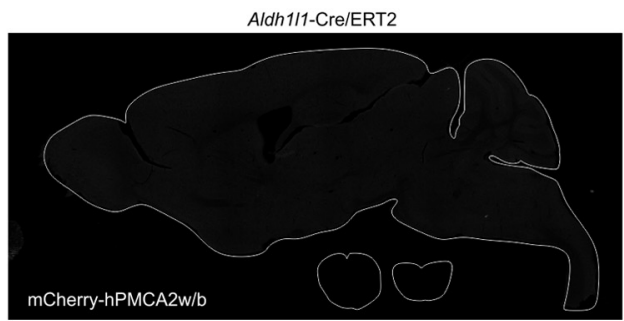

CalEx ${ }^{\text {haxwill }}$ Aldh1/1-Cre/ERT2 (Tamoxifen 75 mg/kg x 1)

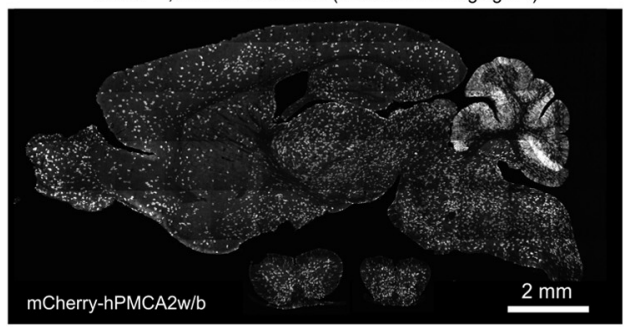

B

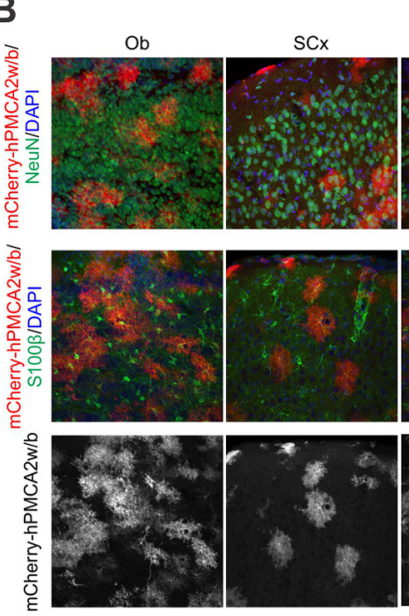

CalEx ${ }^{\text {froximl }} ;$ Aldh1/1-Cre/ERT2 (Tamoxifen $75 \mathrm{mg} / \mathrm{kg} \times 5$ )
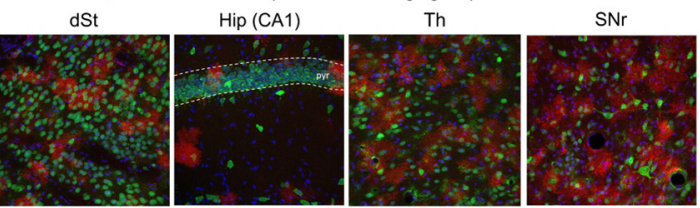

$\mathrm{Cb}$

C
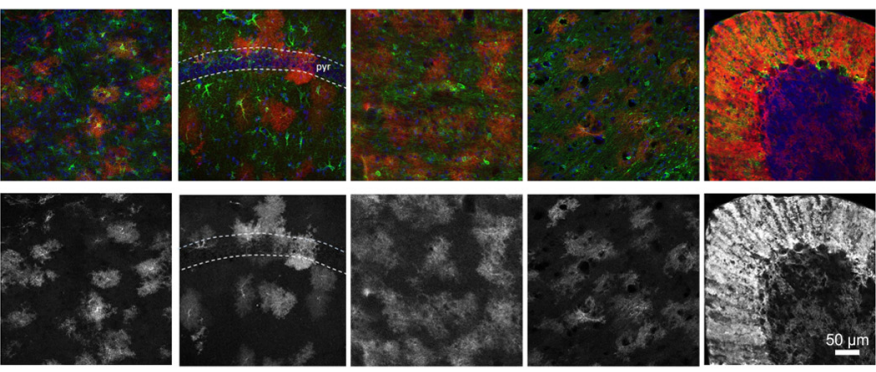

CalEx ${ }^{\text {noxwm }}$; Aldh1/1-Cre/ERT2 (Tamoxifen $75 \mathrm{mg} / \mathrm{kg} \times 1$ )
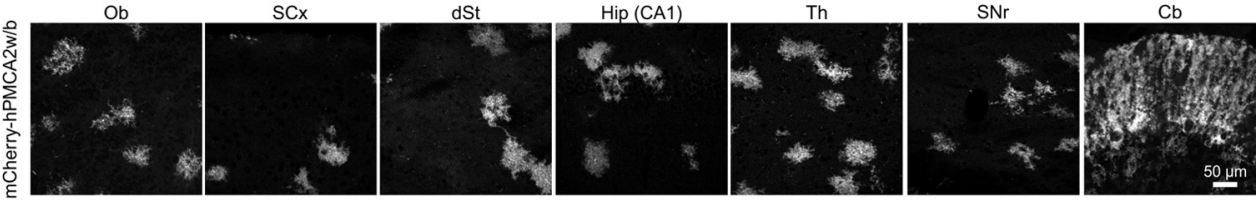

D

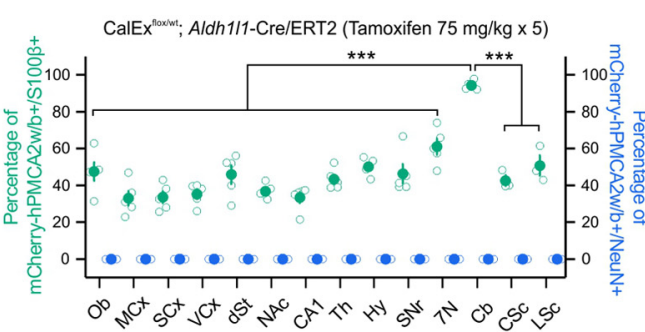

E

CalEx ${ }^{\text {noxmm }} ;$ Aldh111-Cre/ERT2 (Tamoxifen 75 mg/kg x 1)

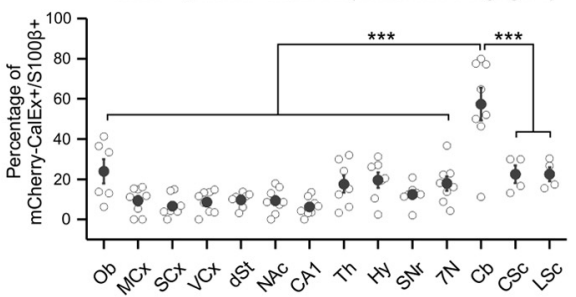

Figure 4. Expression of mCherry-hPMCAw2/b was the greatest in the cerebellum of bigenic mice. $A, I H C$ images of the sagittal sections of the entire brain and coronal sections of the spinal cord showing a global expression of mCherry-hPMCA2w/b in bigenic mice that received tamoxifen but not in control mice (CalEx ${ }^{\text {flox }}$ or Aldh1/1-Cre/ERT2 mice). $\boldsymbol{B}$, IHC images of seven brain regions showing expression of $\mathrm{mCherry}-\mathrm{hPMCA2w/b}$ in bigenic mice with multiple doses of tamoxifen injection (bottom). mCherry-hPMCA2w/b expression colocalized with astrocyte marker $5100 \beta$ (middle) but not neuronal marker NeuN (top). C, IHC images of seven brain regions showing expression of mCherry-hPMCA2w/b in bigenic mice with single dose tamoxifen injection. $\boldsymbol{D}$, Quantification of the percentage of $\mathrm{S} 100 \beta+$ cells and NeuN + cells that colocalized with mCherry-hPMCA2w/b across 14 CNS regions in bigenic mice with multiple doses of tamoxifen injection. Compared with other areas, the cerebellum has the highest percentage of $S 100 \beta+$ cells expressing mCherry-hPMCA2w/b ( $n=3-5$ mice per region, $F_{(13,52)}=20.01, p<0.0001$, one-way ANOVA with a post hoc Bonferroni test). No neuronal expression was detected. $\boldsymbol{E}$, The percentage of $\mathrm{S100} \beta+$ cells colocalized with $\mathrm{mCherry}$-hPMCA2w/b is the greatest in the cerebellum across 14 CNS regions in bigenic mice with single dose tamoxifen injection ( $n=4-8$ mice per region, $F_{(13,85)}=13.18, p<0.0001$, one-way ANOVA with a post hoc Bonferroni test). Ob, olfactory bulb; $M C x$, motor cortex; SCX, sensory cortex; VCX, visual cortex; dSt, dorsal striatum; NAc, nucleus accumbens; CA1, hippocampal CA1; Th, thalamus; Hy, hypothalamus; SNr, substantia nigra; 7N, facial motor nucleus; Cb, cerebellum; CSC, cervical spinal cord; $L S c$, lumbar spinal cord. The average data are shown as mean \pm SEM. In some cases, the SEM symbol is smaller than the symbol for the mean; $* * * p<0.001$. 
Table 1. Expression of $m$ Cherry-hPMCA2w/b in 14 CNS regions of bigenic mice

\begin{tabular}{|c|c|c|c|c|}
\hline \multirow[b]{2}{*}{$\begin{array}{l}\text { CNS regions (rostral } \\
\text { to caudal) }\end{array}$} & \multicolumn{2}{|c|}{ Multiple (5) tamoxifen $75 \mathrm{mg} / \mathrm{kg}$, i.p. injections } & \multicolumn{2}{|l|}{ Single tamoxifen 75 mg/kg, i.p. injection } \\
\hline & $\begin{array}{l}\text { Percentage of mCherry-hPMCA2w/b+ } \\
\text { and } 5100 \beta+(\%)\end{array}$ & $\begin{array}{l}\text { mCherry-hPMCA2w/b intensity } \\
\text { per cell (a.u.) }\end{array}$ & $\begin{array}{l}\text { Percentage of mCherry-hPMCA2w/b + } \\
\text { and } \mathrm{S} 100 \beta+(\%)\end{array}$ & $\begin{array}{l}\text { mCherry-hPMCA2w/b intensity } \\
\text { per cell (a.u.) }\end{array}$ \\
\hline$M C x$ & $33.0 \pm 4.1$ & $77.1 \pm 4.0$ & $9.3 \pm 2.4$ & $29.9 \pm 3.1$ \\
\hline SCx & $33.5 \pm 3.2$ & $81.6 \pm 3.4$ & $6.6 \pm 1.9$ & $29.6 \pm 3.0$ \\
\hline$V C x$ & $35.3 \pm 2.7$ & $76.1 \pm 3.5$ & $8.6 \pm 2.0$ & $34.6 \pm 2.3$ \\
\hline CA1 & $33.3 \pm 3.0$ & $62.2 \pm 2.5$ & $6.3 \pm 1.6$ & $25.1 \pm 1.8$ \\
\hline Th & $43.3 \pm 2.5$ & $60.4 \pm 1.8$ & $17.6 \pm 4.3$ & $21.5 \pm 1.0$ \\
\hline Hy & $50.1 \pm 2.1$ & $68.5 \pm 2.3$ & $19.6 \pm 3.8$ & $24.8 \pm 1.1$ \\
\hline $\mathrm{SNr}$ & $46.3 \pm 5.2$ & $62.0 \pm 2.4$ & $12.4 \pm 2.1$ & $26.5 \pm 1.1$ \\
\hline $7 \mathrm{~N}$ & $61.1 \pm 4.3$ & $65.0 \pm 2.1$ & $17.8 \pm 3.5$ & $31.3 \pm 1.4$ \\
\hline $\mathrm{Cb}$ & $94.3 \pm 1.1$ & $134.5 \pm 3.7$ & $57.4 \pm 8.1$ & $75.1 \pm 1.8$ \\
\hline
\end{tabular}

The data are reported from between three and eight mice in each case.

mice per region, $F_{(13,52)}=20.01, p<0.0001$, one-way ANOVA with a post hoc Bonferroni test; Fig. $4 D$; Table 1) with multiple tamoxifen doses. No NeuN+ cells expressed mCherryhPMCA2w/b (Fig. 4B,D). The proportion of astrocytes expressing $\mathrm{mCherry-hPMCA} 2 \mathrm{w} / \mathrm{b}$ in the bigenic mice was lower overall with a single dose of tamoxifen, however the cerebellum still showed significantly higher expression ( $9 \%$ in $\mathrm{MCx}$ vs $57 \%$ in $\mathrm{Cb}, n=4-8$ mice per region, $F_{(13,85)}=13.18, p<0.0001$, one-way ANOVA with a post hoc Bonferroni test; Fig. 4E; Table 1). Furthermore, the intensity of mCherry-hPMCA2w/b fluorescence in individual cells from all 14 CNS regions was also the strongest in Bergmann glia of the $\mathrm{Cb}$ in bigenic mice with multiple tamoxifen doses $(n=69-148$ cells per region from 3-5 mice, $F_{(13,1439)}=65.04, p<0.0001$, one-way ANOVA with a post hoc Bonferroni test; Table 1$)$ and with a single dose $(n=27-129$ cells per region from $4-8$ mice, $F_{(13,753)}=100.49, p<0.0001$, one-way ANOVA with a post hoc Bonferroni test; Table 1). There was no detectable change in tissue architecture in any brain region (Fig. $5 A$ ) and there was no expression of mCherry-hPMCA2w/b in peripheral organs except low levels in the small intestine (Fig. $5 B$, $C)$. Thus, the data suggest that the high and strong mCherryhPMCA2w/b expression in Bergmann glia of the $\mathrm{Cb}$ is the most parsimonious explanation for the motor abnormalities that were observed (Fig. 3).

$\mathrm{Ca}^{2 \pm}$ signaling attenuation in Bergmann glia by mCherry$h P M C A 2 w / b$. To evaluate whether mCherry-hPMCA2w/b expression affects $\mathrm{Ca}^{2+}$ signaling in Bergmann glia, we microinjected AAV2/5-G $f a A B C_{1} D$-GCaMP6f together with either the control or Cre AAVs into the cerebellum of CalEx ${ }^{\text {flox }}$ mice and performed $\mathrm{Ca}^{2+}$ imaging on brain slices two to three weeks later (Fig. 6A). This approach permitted co-expression of GCaMP6f and mCherry-hPMCA2w/b in Bergmann glia (Fig. 6B,C). The expression of mCherry-hPMCA2w/b was substantial in Bergmann glia processes that extended through the molecular layer (Fig. 6C). It has been reported that Bergmann glia express various GPCRs that couple to $\mathrm{Ca}^{2+}$ release from intracellular stores, including $\alpha 1$-adrenoceptors and $\mathrm{P} 2$ purinergic receptors (Kulik et al., 1999; Piet and Jahr, 2007). Consistently, bath application of GPCR agonists (10 $\mu \mathrm{M}$ PE or $100 \mu \mathrm{M}$ ATP) triggered robust $\mathrm{Ca}^{2+}$ increases in Bergmann glia of CalEx ${ }^{\text {flox }}$ mice that received the control AAV (Fig. 6D-I). Expression of mCherryhPMCA2w/b significantly attenuated $10 \mu \mathrm{M}$ PE-evoked $\mathrm{Ca}^{2+}$ responses by $\sim 90 \%$ in the somata of Bergmann glia $(n=39-67$ cells from 4-6 mice per group, $U=2602$, $p<0.0001$, MannWhitney test; Fig. 6E,F). A similar effect was observed with ATP application and the $\mathrm{Ca}^{2+}$ responses of Bergmann glia were reduced by $\sim 80 \%$ in CalEx ${ }^{\text {flox }}$ mice with the Cre AAV $(n=26-$ 55 cells from 5 mice per group, $U=1413, p<0.0001$, MannWhitney test; Fig. 6H,I).

\section{Cerebellar transcriptomic changes in multiple cell types by} Bergmann glia $\mathrm{Ca}^{2 \pm}$ attenuation

To explore the molecular alterations induced by Bergmann glia $\mathrm{Ca}^{2+}$ attenuation on whole cerebellar tissue in an unbiased manner, we employed RNA-seq to profile the transcriptomic changes in bigenic mice (Fig. 7A). Five days after a single dose of tamoxifen, RNA was extracted from the cerebella of $\mathrm{CalEx}^{\text {flox/wt }}$ mice (control) and bigenic mice for sequencing and subsequent analyses $(n=4$ mice per group; Fig. $7 A)$. Comparing the bulk RNAseq data between bigenic and control mice, we identified 7923 differentially expressed genes (DEGs) in the Cb, with 4146 DEGs downregulated and 3777 DEGs upregulated (FPKM $>0$, fold change $>1$, FDR $<0.05$; Fig. $7 B$; Extended Data Table 7-1). Taking the transcript abundance into consideration, we further applied different thresholds including the level of expression (FPKM) and the degree of change (fold change; Fig. 7B). By assigning FPKM of 5 and fold change of 2, there were 314 DEGs detected, with 147 downregulated DEGs and 167 upregulated DEGs. We next evaluated the top 50 DEGs ranked by the degree of expression change induced by Bergmann glia $\mathrm{Ca}^{2+}$ attenuation, which ranged from 468-fold decrease (Ttr) to 16-fold increase $(L c n 2$; FPKM $>5$, FDR $<0.05$; Fig. $7 C)$. The biological functions that are associated with these profound cerebellar changes included ion transport, apoptotic process, transcription regulation, endocytosis and cell proliferation/growth/migration (Fig. 7C). Among the top 50 DEGs, the most upregulated gene was $L c n 2$, which is considered as a pro-inflammatory factor and a marker gene for reactive astrocytes. To examine whether Bergmann glia $\mathrm{Ca}^{2+}$ attenuation triggered astrogliosis, we assessed the expression of a panel of 38 genes containing pan reactive, A1-specific and A2-specific markers that identify reactive astrocytes (Liddelow et al., 2017). Compared with positive control of neuroinflammation using lipopolysaccharide (LPS), the changes in these 38 reactive astrocyte markers induced by 
A

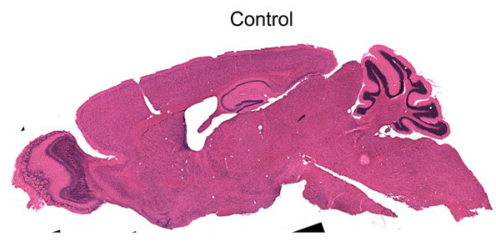

B

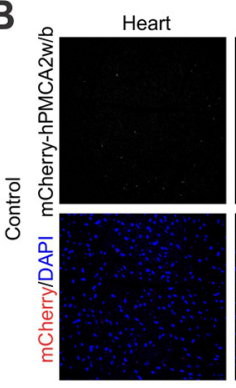

C

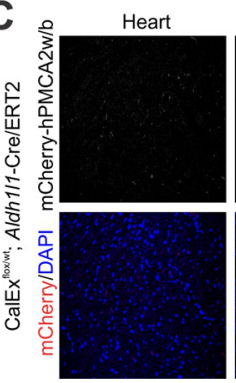

Lungs

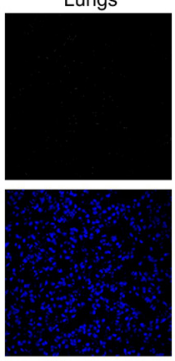

Lungs

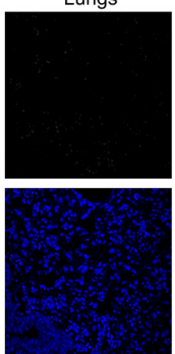

Liver

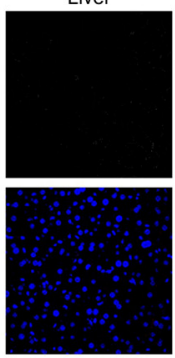

Liver

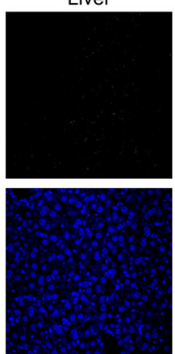

CalEx ${ }^{\text {noxwm; }} ;$ Aldh1/1-Cre/ERT2

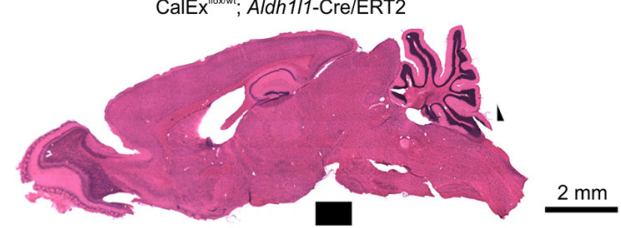

Spleen

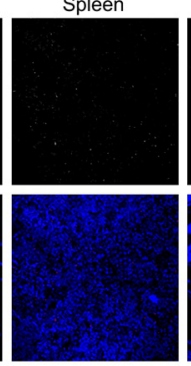

Small intestine
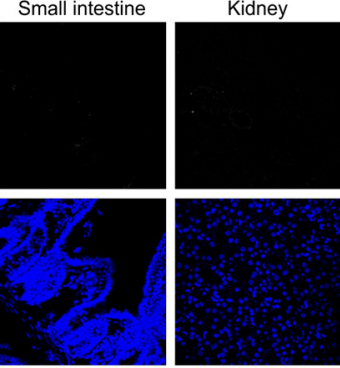

Kidney
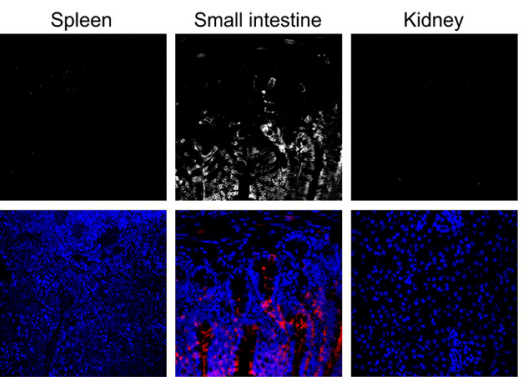

Muscle
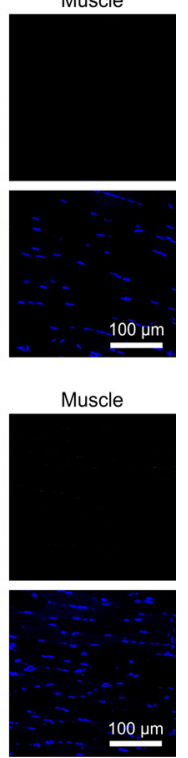

Figure 5. Expression of mCherry-hPMCA2w/b in peripheral organs of bigenic mice with the multiple dose tamoxifen protocol. $\boldsymbol{A}$, Hematoxylin and eosin staining showing that the gross histology of the brain from bigenic (CalEx flox/wt; Aldh1/7-Cre/ERT2) mice was not altered. B, C, IHC images showing mCherry-hMPCA2w/b expression from seven peripheral organs including the heart, lungs, liver, spleen, small intestine, kidney, and muscle from the control $(\boldsymbol{B})$ and bigenic $(\boldsymbol{C})$ mice. mCherry-CalEx expression was detected in the small intestine but not in other organs.

Bergmann glia $\mathrm{Ca}^{2+}$ attenuation was moderate (Fig. 7D). Specifically, the average increase across all markers was $3.3 \pm 1.4$-fold in the $\mathrm{Cb}$ of bigenic mice versus $19.2 \pm 5.7$-fold in LPS. Based on identified gene expression changes (FPKM $>1$, FDR $<0.05$ ), we summarized the top 10 canonical pathways that were significantly altered in the cerebellar tissue $(p<0.05$; Fig. $7 E$ ). Nine out of ten pathways were found to be activated by Bergmann glia $\mathrm{Ca}^{2+}$ attenuation, including senescence pathway, synaptogenesis signaling pathway and stress response. In contrast, a signaling pathway mediated by PTEN tumor suppressor (Lee et al., 2018) was significantly inhibited. Thus, Bergmann glia $\mathrm{Ca}^{2+}$ attenuation in bigenic mice resulted in pronounced changes in the whole cerebellar tissue for both genes and signaling pathways.

Similar to other brain regions, the $\mathrm{Cb}$ is composed of different cell types. We next investigated how Bergmann glia $\mathrm{Ca}^{2+}$ attenuation affects various cell populations by integrating the bulk RNA-seq data with previously published single-cell RNA-seq (scRNA-seq) data of the adult mouse $\mathrm{Cb}$ (Zeisel et al., 2018). The Cb scRNA-seq generated transcriptomic profiles of 11,063 cells and identified 11 transcriptomic clusters corresponding to known major cerebellar cell types. We used the top 1000 genes for six most dominant cerebellar cell types as a reference and mapped DEGs identified from bulk RNA-seq (FPKM $>1$, FDR $<0.05)$ to explore the cell-type-specific alterations by Bergmann glia $\mathrm{Ca}^{2+}$ attenuation (Fig. $7 F$ ). The highest proportion of DEGs was mapped to neurons $(\sim 37.8 \%)$, followed by astrocytes $(\sim 17.4 \%)$, microglia $(\sim 16.7 \%)$, oligodendrocytes $(\sim 10.7 \%)$, ependyma $(\sim 8.8 \%)$, and fibroblast-like cells $(\sim 8.6 \%)$. Specifically, Purkinje cells were the most affected neuronal populations, with $\sim 17 \%$ DEGs mapped. This result is in accordance with the close proximity between Bergmann glia and Purkinje cells as well as their communications via Bergmann glia $\mathrm{Ca}^{2+}$ signals. Together, these data suggest global modification of the molecular identities of diverse cells in the $\mathrm{Cb}$ by Bergmann glia $\mathrm{Ca}^{2+}$ attenuation. We suggest such changes are the likely cause of the behavioral alterations we observed and together the data show strong consequences of CalEx expression in astrocytes. We emphasize that our data do not explain why the mice die prematurely; this is problematic to address with brain wide expression of CalEx. Instead, the experiments provide plausible hypotheses for the motor deficits that were observed and suggest cerebellar defects as a likely cause.

\section{Mechanistic interpretations and considerations}

The use of CalEx ${ }^{\text {flox }}$ mice resulted in clearly attenuated astrocyte intracellular $\mathrm{Ca}^{2+}$ signaling (Figs. 2, 6), triggered behavioral alterations (Figs. 2, 3), and induced transcriptomic changes (Fig. 7). How should one interpret and potentially connect these findings across scales? We interpret these findings in the context of three descriptive models, which are independent, but non-exclusive (Fig. 8). In the first model, CalEx acts as it was intended - as $\mathrm{a} \mathrm{Ca}^{2+}$ extruder on the plasma membrane of astrocytes to reduce intracellular $\mathrm{Ca}^{2+}$ signaling (Yu et al., 2018). This would reduce $\mathrm{Ca}^{2+}$-dependent processes within astrocytes and also alter $\mathrm{Ca}^{2+}$ handling within the cells, which is orchestrated by operations of several $\mathrm{Ca}^{2+}$ sources including store-operated $\mathrm{Ca}^{2+}$ channels (SOCs) and $\mathrm{Ca}^{2+}$ pumps (PMCAs) located on the plasma membrane, as well as $\mathrm{IP}_{3}$ receptors and SERCA pumps located on the ER (Fig. 8A). Such interpretations are consistent with published data using CalEx, whereby $\mathrm{Ca}^{2+}$ handling resulting from intracellular $\mathrm{Ca}^{2+}$ store depletion was significantly altered (Yu et al., 
A

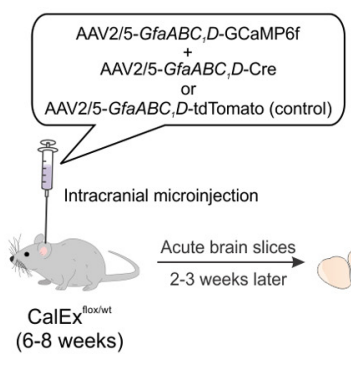

D

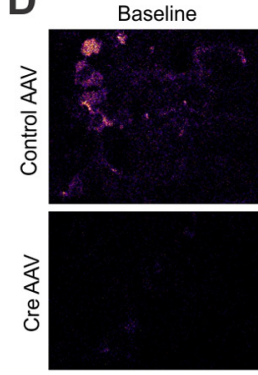

G

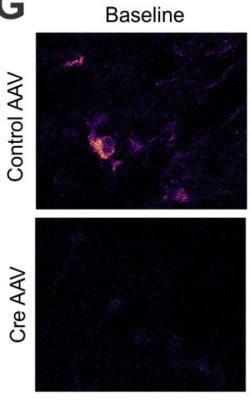

Phenylephrine

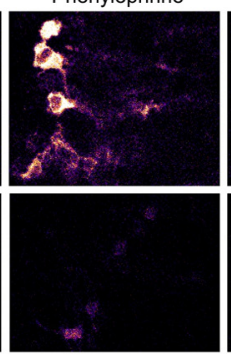

ATP

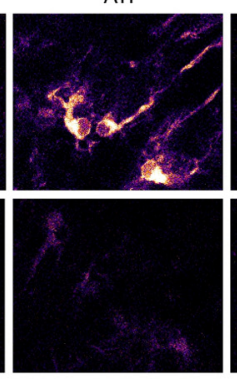

Two-photon $\mathrm{Ca}^{2+}$ imaging

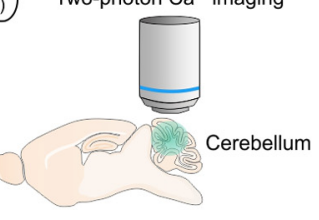

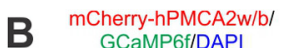

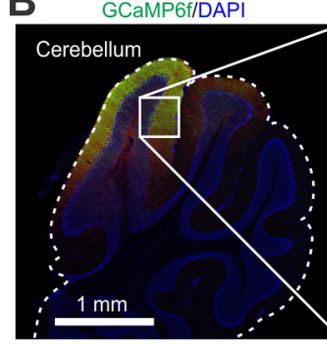

C

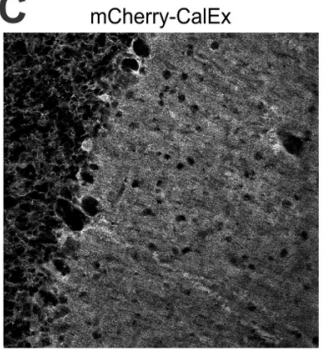

mCherry-hPMCA2w/b/ GCaMP6f/S100ß/DAP

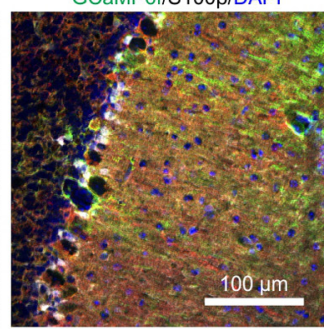

E

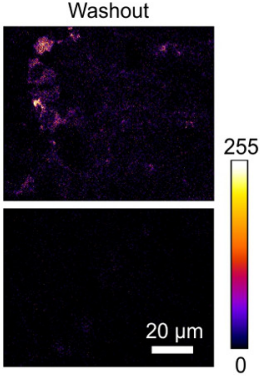

Washout

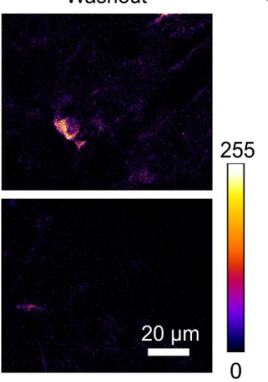

H
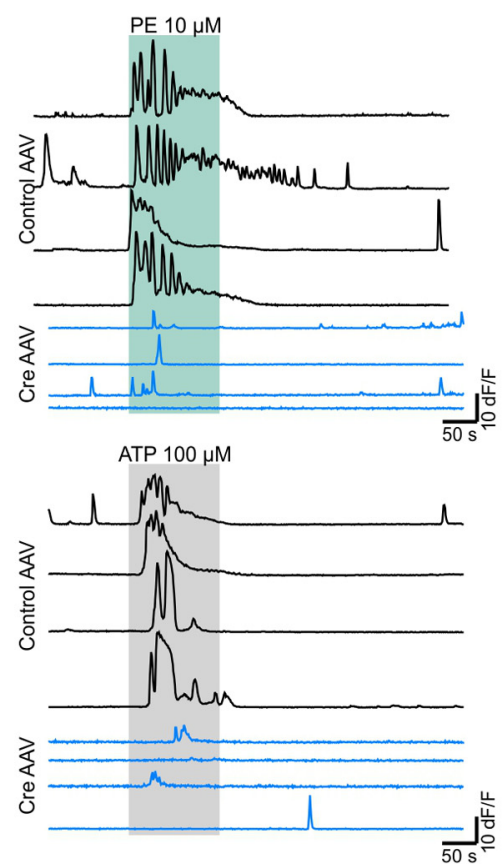

F

Control AAV ( $n=4$ mice, 39 cells) Cre AAV ( $n=6$ mice, 67 cells)

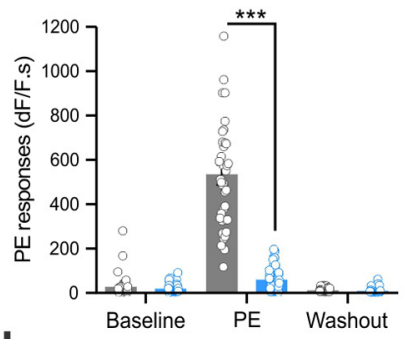

I

Control AAV ( $\mathrm{n}=5$ mice, 26 cells) Cre AAV ( $n=5$ mice, 55 cells)

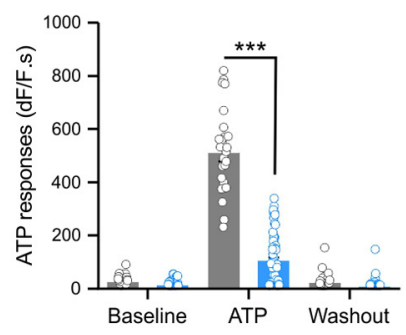

Figure 6. Attenuation of GPCR-mediated $\mathrm{Ca}^{2+}$ signaling in Bergmann glia with CalEx ${ }^{\text {flox }}$ mice. A, Schematic of experimental design. To image $\mathrm{Ca}^{2+}$ signals in Bergmann glia, CalEx ${ }^{\text {flox }}$ mice received microinjections of AAV2/5-GfaABC ${ }_{1} D-G C a M P 6 f$ together with control or Cre AAVs. Two-photon $\mathrm{Ca}^{2+}$ imaging of GCaMP6f-expressing Bergmann glia was performed two to three weeks after the AAV injection. $B, A n I H C$ image of the entire cerebellum showing the expression of GCaMP6f and mCherry-hPMCA2w/b. C, Higher-magnification IHC images showing mCherryhPMCA2w expression in Bergmann glia that was colocalized with GCaMP6f and $\mathrm{S100} \beta$. D, Z-stack images of two-photon $\mathrm{Ca}^{2+}$ imaging of Bergmann glia from CalEx ${ }^{\text {flox }}$ mice microinjected with control (top) or Cre (bottom) AAVs during baseline, PE (10 $\mu \mathrm{M})$ application, and washout. $\boldsymbol{E}$, Representative traces of $\mathrm{Ca}^{2+}$ responses to PE in the somata of Bergmann glia from CalEx ${ }^{\text {flox }}$ mice with control (top) or Cre (bottom) AAVs. $\boldsymbol{F}$, Quantification of PE-induced $\mathrm{Ca}^{2+}$ responses as integrated areas during baseline, PE application, and washout. G, Z-stack images of Bergmann glia from CalEx ${ }^{\text {flox }}$ mice microinjected with control (top) or Cre (bottom) AAVs during baseline, ATP (100 $\left.\mu \mathrm{m}\right)$ application, and washout. $\boldsymbol{H}$, Representative traces of $\mathrm{Ca}^{2+}$ responses to ATP in the somata of Bergmann glia from CalEx ${ }^{\text {flox }}$ mice with control (top) or Cre (bottom) AAVs. I, Quantification of ATP-induced $\mathrm{Ca}^{2+}$ responses as integrated areas during baseline, ATP application, and washout. Average data are shown as mean $\pm \mathrm{SEM} ; * * * p<0.001$.

2018). Furthermore, attenuated $\mathrm{Ca}^{2+}$ signaling in astrocytes by CalEx may induce transcriptional and translational modifications that lead secondarily to synaptic and circuit level changes and ultimately altered behaviors (Yu et al., 2018). This model thus includes two components: primary effects because of altered dynamics of $\mathrm{Ca}^{2+}$ signaling and secondary effects dues to changes in $\mathrm{Ca}^{2+}$-dependent gene expression. Such interpretations align with the fact that $\mathrm{Ca}^{2+}$ ions serve as second messengers for numerous essential physiological functions within cells over a wide range of spatiotemporal scales (Clapham, 2007) and are consistent with the breadth of data on astrocyte $\mathrm{Ca}^{2+}$ signaling (Volterra et al., 2014; Bazargani and Attwell, 2016; Shigetomi et al., 2016). The second model proposes an effect of CalEx on gene expression in astrocytes that is independent of intracellular $\mathrm{Ca}^{2+}$ signaling (Fig. 8B). Although there is no evidence supporting PMCA's direct modulation of gene expression, one possible scenario how this could occur is that CalEx alters the expression of endogenous PMCA and its associated modulators such as cAMP and phosphoinositide 3-kinase (Kuo et al., 1993; Abramowitz et al., 2000), which may conceivably drive changes in signal transduction and gene regulation that contribute to some of the metrics reported in this study. In the third model, CalEx exports sufficient astrocytic intracellular $\mathrm{Ca}^{2+}$ into the extracellular space such that this additional $\mathrm{Ca}^{2+}$ directly alters synaptic transmission and electrical properties of neurons, which then contribute to subsequent behavioral outcomes (Fig. 8C). We cannot rule out this scenario, but consider the likelihood to be relatively low. The pool of extracellular $\mathrm{Ca}^{2+}$ concentration is substantially larger ( $\sim 10^{4}$ times) than that of intracellular $\mathrm{Ca}^{2+}$ levels within astrocytes (Shigetomi et al., 2016) and so the additional change by CalEx, if it occurred, would be moderate and dissipated quickly by diffusion. In addition, neither fast inhibitory or excitatory synaptic transmission onto medium spiny neurons was affected by CalEx expression in striatal astrocytes (Yu et al., 2018). This is of note, because neurotransmitter release probability has a very steep dependency on $\mathrm{Ca}^{2+}$ concentration and would be expected to change if extracellular $\mathrm{Ca}^{2+}$ changed meaningfully. It also seems reasonable to suggest that the behavioral results reported by us may reflect a combination of all three models, which are as of yet hard to tease apart. Of the three 
A

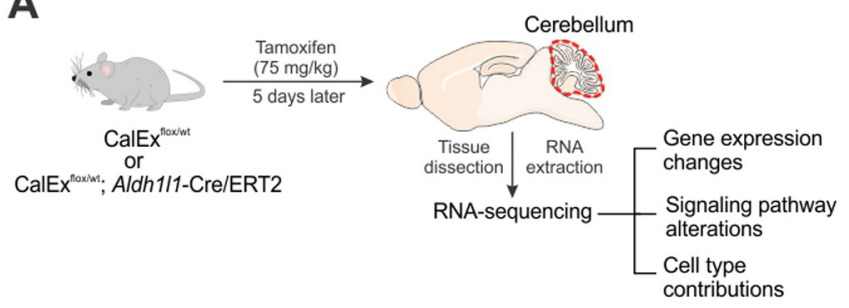

B

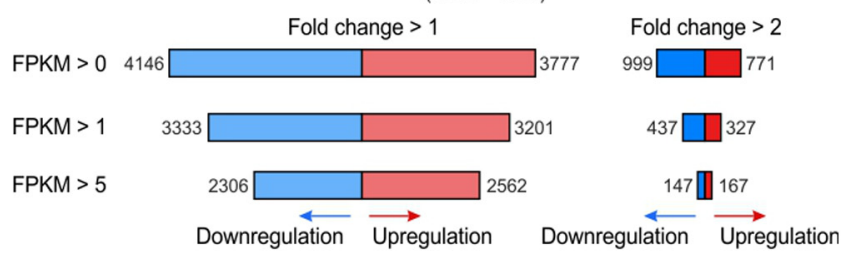

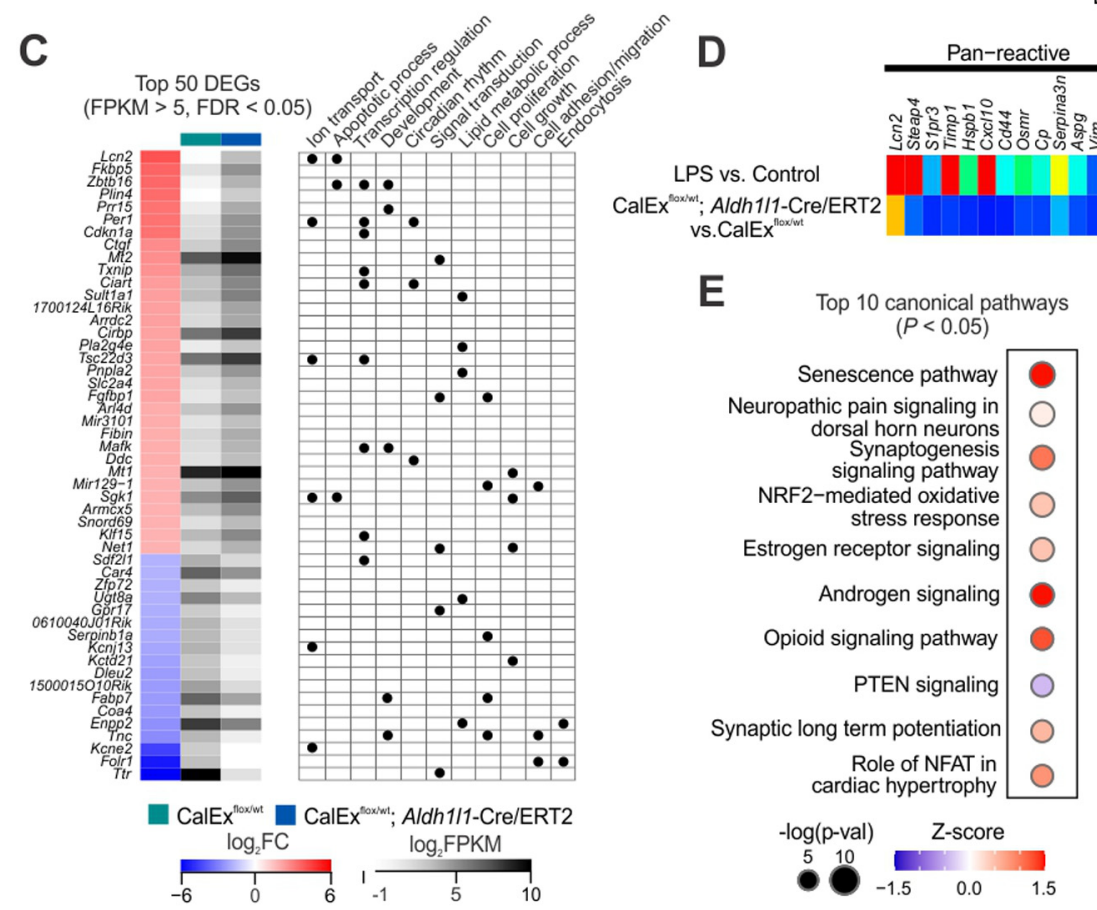

A1-specific A2-specific

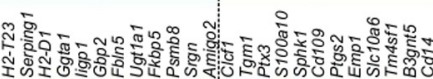
Fold change

$\mathbf{F}$

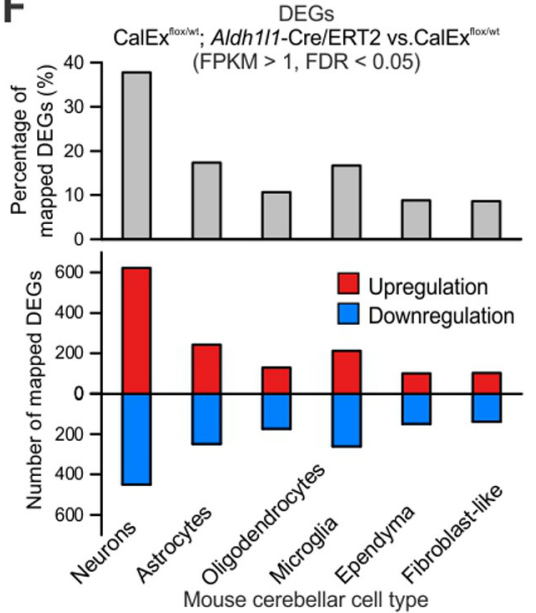

Figure 7. Cerebellar RNA-seq reveals transcriptomic alterations induced by mCherry-hPMCA2w/b expression in bigenic mice. $A$, Schematic of experimental design. Both CalEx ${ }^{\text {flox }}$ mice (control) and bigenic mice received a single dose tamoxifen injection $(75 \mathrm{mg} / \mathrm{kg} \times 1 \mathrm{~d})$ and cerebellar tissues were dissected out and processed for RNA-seq $5 \mathrm{~d}$ later. $B$, Numbers of DEGs (analyzed by edgeR, FDR $<0.05$ ) that were upregulated and downregulated under different thresholds (FPKM $>0,1$ and 5; fold change $>1$ and 2). C, left, Top 50 DEGs (FPKM $>5$, FDR $<0.05$ ) by mCherry-hPMCA2w/b expression in the cerebella of bigenic mice, ranked by fold change when comparing with CalEx ${ }^{\text {flox }}$ mice. Right, Summary table of selected gene ontology terms (biological processes) that are associated with each DEG. $\boldsymbol{D}$, Heat map showing relative expression levels of 38 known reactive astrocyte markers including proposed pan-reactive, A1-specific and A2-specific genes in bigenic mice compared with control mice. $\boldsymbol{E}$, Top 10 altered canonical pathways in cerebellar RNA-seq from bigenic mice. Sizes of dots represent log-transformed $p$ value and colors indicate either pathway activation (red) or inhibition (blue) based on $z$ scores. $\boldsymbol{F}$, top, Percentage of DEGs in bigenic mice (FPKM $>1$, FDR $<0.05$ ) that were mapped onto mouse cerebellar scRNA-seq data, based on the top 1000 cell type marker genes for the six major cell types. Bottom, Numbers of mapped DEGs onto different cerebellar cell types that were upregulated and downregulated. List of DEGs identified in the cerebellar RNA-seq of bigenic mice are listed in Extended Data Table 7-1.

models, the first one is informative from the perspective of astrocyte biology, but the latter two may produce coincidental effects that are significant, but nonetheless not reflective of astrocyte biology in a physiological sense. We have recently discussed the necessity to assess astrocyte findings by proposing three types of interpretations (Nagai et al., 2021). Within that framework, the first model represents a type 1 or type 2 interpretation, whereas the second and third models represents an indirect type 3 interpretation (Nagai et al., 2021). Our findings, and those in the future with the use of CalEx, should be interpreted cautiously from the perspective of multiple models to account for the empirical data across scales. Additional tools and approaches are needed to dissect astrocyte signaling more precisely.

\section{Discussion}

There are three main findings from this study. First, we provide a genetic tool to attenuate $\mathrm{Ca}^{2+}$ signals using knock-in CalEx ${ }^{\text {flox }}$ mice at the Rosa 26 locus. In these mice, mCherry-hPMCA2w/b can be expressed in a Cre-dependent manner in genetically specified cells for which a Cre mouse line or Cre-expressing virus exists. mCherry-hPMCA2w/b expression was controlled by Cre expression and caused $\sim 90 \%$ attenuation of $\mathrm{Ca}^{2+}$ signaling in astrocytes. CalEx ${ }^{\text {flox }}$ mice can be used to explore contributions of astrocyte $\mathrm{Ca}^{2+}$ signaling in a brain-region-specific manner with local Cre AAV microinjections or with region-specific astrocyte Cre lines as they are developed. Our data provide validation of CalEx ${ }^{\text {flox }}$ mice, but do not obviate the need for further controls when the mice are used in future studies. This is important to consider since astrocytes are a heterogeneous population of cells and thus specific controls will be needed on a case-by-case basis for particular brain regions and Cre lines (Haim and Rowitch, 2017; Khakh and Deneen, 2019; Pestana et al., 2020). The necessity of experiments tailored to specific brain regions is exemplified by recent insights on the brain region-specific regulation of astrocyte function by transcription factors (Huang et al., 2020). Second, we explored the consequences of CalEx expression in astrocytes in a CNS-wide manner and observed profound alterations in mouse behavior and premature lethality. Third, we focused on the cerebellum based on the histologic analysis and 


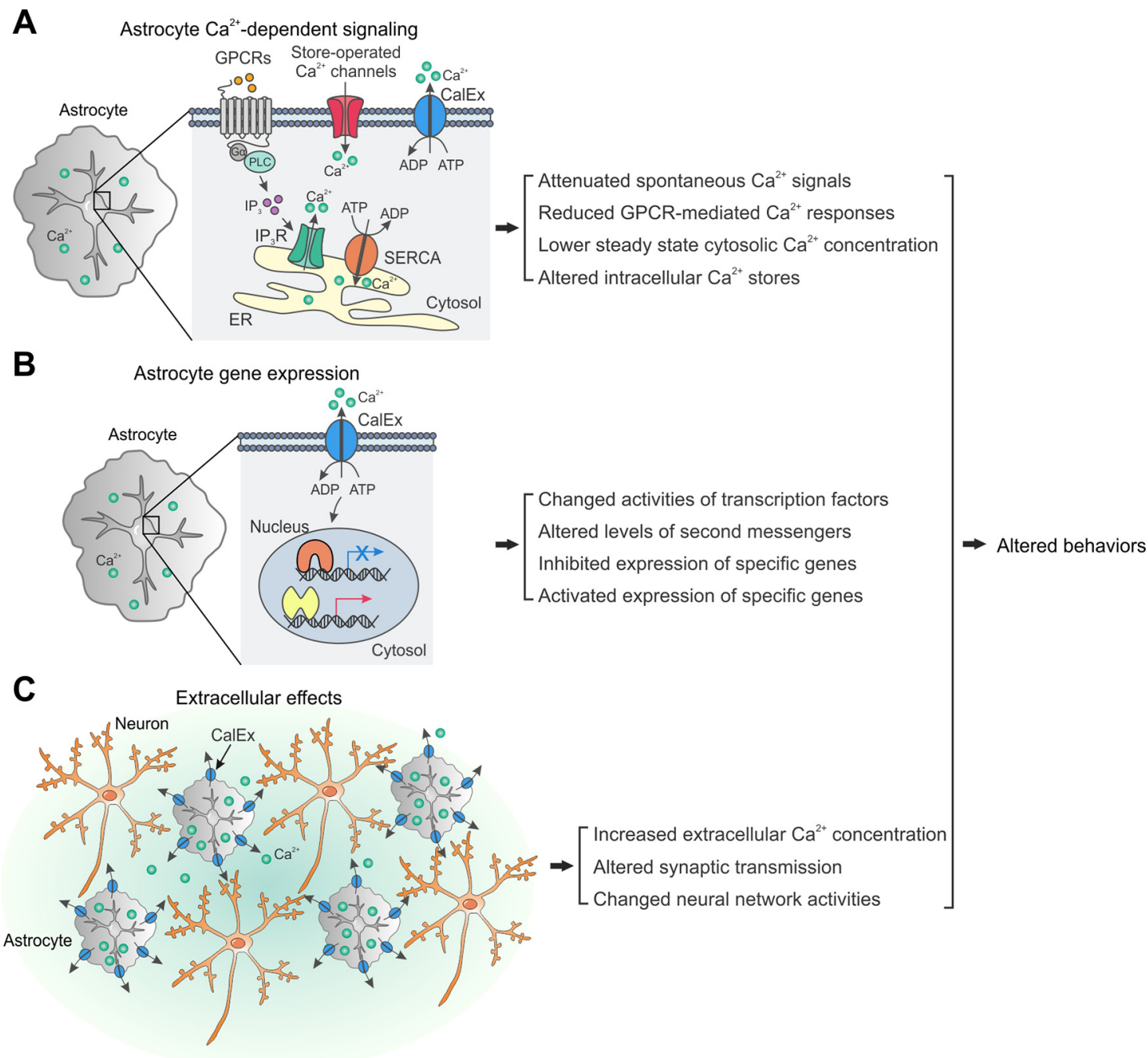

Figure 8. Schematics to illustrate descriptive models of astrocyte CalEx-induced effects that are reported in this study. $\boldsymbol{A}$, Astrocyte $\mathrm{Ca}^{2+}$-dependent signaling. Extrusion of $\mathrm{Ca}^{2+}$ by $\mathrm{CalEx}_{\mathrm{E}}$ in astrocytes alters intracellular $\mathrm{Ca}^{2+}$ handling. The major $\mathrm{Ca}^{2+}$ sources in astrocytes include SOCs and various calcium-permeable pumps located on the plasma membrane, $\mathrm{IP}_{3}$ receptors, and SERCA pumps located on the ER. $\boldsymbol{B}$, Astrocyte gene expression. CalEx expression in astrocytes triggers transcriptional changes that are independent of intracellular $\mathrm{Ca}^{2+}$ signaling through a currently unknown mechanism. C, Extracellular effects. CalEx expression in astrocytes exports sufficient amount of $\mathrm{Ca}^{2+}$ ions into the extracellular space to alter synaptic transmission and neural circuit activity. Some combination of all three models could operate in parallel.

identified molecular changes at the gene and pathway levels of multiple cell types that were associated with CalEx in Bergmann glia.

Several biological responses have been ascribed to astrocytes and are proposed to occur via intracellular $\mathrm{Ca}^{2+}$ signaling. These include regulation of neurons, contributions to diverse diseases, and control of blood flow (Araque et al., 2014; Howarth, 2014; Volterra et al., 2014; Khakh and McCarthy, 2015; Bazargani and Attwell, 2016; Verkhratsky and Nedergaard, 2018). CalEx ${ }^{\text {flox }}$ mice could be used to study such responses in vivo for genetically defined cell populations, e.g., $\mathrm{Ca}^{2+}$-dependent astrocyte contributions to motor function, olfactory responses, learning, circadian rhythms, and drug-evoked behaviors, as well as others (Nimmerjahn et al., 2009; Lee et al., 2010; Brancaccio et al., 2017; Adamsky et al., 2018; Corkrum et al., 2020; Huang et al., 2020; Ung et al., 2020). Furthermore, although we focused on astrocyte studies, $\mathrm{CalEx}{ }^{\text {flox }}$ mice could be used to explore the consequences of attenuating $\mathrm{Ca}^{2+}$ signaling in other CNS cells including neurons, microglia, pericytes, oligodendrocytes, and endothelial cells for which reliable Cre lines exist. Our studies with bigenic mice show that strongly attenuating astrocyte $\mathrm{Ca}^{2+}$ signaling across the CNS with mCherry-hPMCA2w/b may lead to premature lethality, supporting the proposition that astrocyte $\mathrm{Ca}^{2+}$ may serve multiple critical functions rather than being inconsequential as was thought (Petravicz et al., 2008, 2014; Agulhon et al., 2010). Perhaps the simplest interpretation of premature lethality in the bigenic mice with CNS-wide astrocyte $\mathrm{Ca}^{2+}$ signaling attenuation is that several important responses were impaired (Fig. 8) beyond a critical threshold resulting in loss of essential functions and death, although admittedly we have not been able to determine why the mice died. In these regards, whole body $\mathrm{IP}_{3} \mathrm{R} 2-/-$ mice do not display similar lethality (Petravicz et al., 2008, 2014; Agulhon et al., 2010), but it should be remembered that these mice display residual $\mathrm{Ca}^{2+}$ signaling that persisted after $\mathrm{IP}_{3} \mathrm{R} 2$ deletion (Di Castro et al., 2011; Srinivasan et al., 2015; Rungta et al., 2016; Agarwal et al., 2017). In addition, the deletion of $\mathrm{IP}_{3} \mathrm{R} 2$ throughout development may stimulate compensatory mechanisms that are unlikely to occur in the mature CNS after inducible mCherry-hPMCA2w/b expression. In contrast to $\mathrm{IP}_{3} \mathrm{R} 2$ deficiency, mCherry-hPMCA2w/b has a broader impact on $\mathrm{Ca}^{2+}$ sources and $\mathrm{Ca}^{2+}$-dependent pathways including $\mathrm{IP}_{3}$ independent ones, which should be taken into account when 
interpreting the physiological significance of astrocyte $\mathrm{Ca}^{2+}$ signaling and our data (Fig. 8A). Furthermore, high mCherryhPMCA2w/b expression in the cerebellum likely explains major motor dysfunctions that were observed in the bigenic mice. Our data are therefore broadly consistent with critical functions ascribed to astrocyte $\mathrm{Ca}^{2+}$ signaling such as regulation of multiple cell types, including neurons and neurovascular coupling (Attwell et al., 2010; Araque et al., 2014; Volterra et al., 2014; Khakh and McCarthy, 2015; Bazargani and Attwell, 2016; Shigetomi et al., 2016; Savtchouk and Volterra, 2018), and specific contributions to cerebellar function (Watanabe, 2002; Nimmerjahn et al., 2009; Lee et al., 2010; Nimmerjahn and Bergles, 2015). We also consider alternative explanations that mCherry-hPMCA2w/b expression in astrocytes may trigger transcriptional and translational changes bypassing its $\mathrm{Ca}^{2+}$ regulatory function and that constitutive $\mathrm{Ca}^{2+}$ extrusion by mCherry-hPMCA2w/b may alter extracellular $\mathrm{Ca}^{2+}$ concentration and synaptic transmission (Fig. 8). All three proposed models are independent, but non-exclusive. For instance, it is conceivable that altered intracellular $\mathrm{Ca}^{2+}$-dependent signaling by CalEx may further induce transcriptional and posttranscriptional modifications of specific genes that have essential physiological functions. These mechanisms could be explored using $\mathrm{CalEx}^{\text {flox }}$ mice in a brain region-specific manner with specific hypothesis-driven experiments. In these regards, the CalEx ${ }^{\text {flox }}$ mice are a valuable addition to the toolbox available to explore astrocyte biology (Yu et al., 2020a).

We propose several interpretations of how CalEx influences astrocyte function, neuronal and circuit activity based on our experimental observations. The first model considers a causative relationship between astrocyte $\mathrm{Ca}^{2+}$-dependent signaling and behavior with an assumption that the level of $\mathrm{Ca}^{2+}$ signaling attenuation observed in CalEx ${ }^{\text {flox }}$ mice is accounted for by $\mathrm{Ca}^{2+}$ extrusion through hPMCA2w/b pumps. This idea can be theoretically tested by a data-driven mechanistic computational model of astrocyte $\mathrm{Ca}^{2+}$ dynamics in conjunction with $\mathrm{CalEx}{ }^{\text {flox }}$ mice, for instance, with a single-compartment model (Handy et al., 2017; Taheri et al., 2017) or even better with ones that include a spatial dimension (Savtchenko et al., 2018). With further experimental data on astrocyte $\mathrm{Ca}^{2+}$ dynamics and fine structure at sufficient resolution to inform the modeling, underlying $\mathrm{Ca}^{2+}$ fluxes from different astrocyte subcellular regions such as major branches and processes could also be incorporated into the model to capture the complexity of astrocyte morphology and $\mathrm{Ca}^{2+}$ signaling. Moreover, such models could also incorporate astrocyte $\mathrm{Ca}^{2+}$ waves as has been done for cell culture experiments (Roth et al., 1995). Finally, to model astrocyte spatiotemporal $\mathrm{Ca}^{2+}$ dynamics, the single-compartment model could be extended. In order to construct an improved model, realistic constraints need to be obtained empirically using detailed 3D views of astrocyte morphology with high isotropic resolution electron microscopy and time resolved 3D imaging data with methods such as focused ion beam scanning electron microcopy (Xu et al., 2017) and with swept, confocally aligned planar excitation (SCAPE) microscopy (Voleti et al., 2019), respectively. Such data do not currently exist, but are much needed to develop models of astrocyte $\mathrm{Ca}^{2+}$ signals in cells with realistic geometry and dynamics.

We would be remiss if we failed to discuss the limitations of the CalEx approach and opportunities for further improvements in future studies. Compared with the CalEx AAV strategy, $\mathrm{CalEx}^{\text {flox }}$ mice permit more rapid expression of mCherryhPMCA2w/b such that it becomes detectable within days after
Cre AAV microinjections or tamoxifen induction. The CalEx ${ }^{\text {flox }}$ mice also permit greater $\mathrm{Ca}^{2+}$ attenuation than previously possible with CalEx AAVs. However, expression of CalEx as an attenuation tool is still far slower than commonly used actuation approaches such a DREADDs and optogenetic tools which operate in minutes and seconds. Another difference between CalEx and optogenetic and chemogenetic approaches is that $\mathrm{Ca}^{2+}$ extrusion via CalEx is constitutive. In future studies, these issues could be potentially tackled by protein engineering, for instance to make the hPMCA2w/b ligand-activated or light-activated and thus permit temporal control over $\mathrm{Ca}^{2+}$ attenuation. Furthermore, targeting of CalEx to subcellular domains of astrocytes should improve its ability to attenuate distinct forms of $\mathrm{Ca}^{2+}$ signaling selectively. Thus, in our experiments, CalEx expresses broadly on the plasma membrane of astrocytes and its operation does not distinguish between various $\mathrm{Ca}^{2+}$ sources and signaling pathways, which is exemplified by the experiments and the interpretations in this study. Additional modifications could be made to target the pump to specific cellular and subcellular compartments of astrocytes (e.g., somata, end feet and synaptic processes) for refined spatial manipulation of $\mathrm{Ca}^{2+}$ signaling. As we learn more about the transcriptomes and proteomes of astrocytes, such refinements could conceivably be made rationally. Although imperfect, our studies show that CalEx is a useful tool to explore astrocyte biology and portend new developments and improvements.

In summary, our empirical data indicate that astrocyte $\mathrm{Ca}^{2+}$ signaling may serve critical functions for brain function, such that attenuation in a CNS-wide manner resulted in profound behavioral alterations and lethality. The severity of the phenotypes underscores the need to explore astrocyte functions locally in specific brain regions as previously suggested (Yu et al., 2020a), raises awareness of the need for methods to genetically target specific populations of astrocytes in future studies, and highlights the need to candidly consider multiple mechanistic interpretations until such time that more precise experiments are possible (Fig. 8). Furthermore, the mouse we report adds to an emerging toolbox to explore astrocyte biology (Hirbec et al., 2020; Yu et al., 2020a) and will enable new types of experiments to explore astrocytic contributions to CNS function.

\section{References}

Abramowitz J, Aydemir-Koksoy A, Helgason T, Jemelka S, Odebunmi T, Seidel CL, Allen JC (2000) Expression of plasma membrane calcium ATPases in phenotypically distinct canine vascular smooth muscle cells. J Mol Cell Cardiol 32:777-789.

Adamsky A, Kol A, Kreisel T, Doron A, Ozeri-Engelhard N, Melcer T, Refaeli R, Horn H, Regev L, Groysman M, London M, Goshen I (2018) Astrocytic activation generates de novo neuronal potentiation and memory enhancement. Cell 174:59-71.

Agarwal A, Wu PH, Hughes EG, Fukaya M, Tischfield MA, Langseth AJ, Wirtz D, Bergles DE (2017) Transient opening of the mitochondrial permeability transition pore induces microdomain calcium transients in astrocyte processes. Neuron 93:587-605.

Agulhon C, Petravicz J, McMullen AB, Sweger EJ, Minton SK, Taves SR, Casper KB, Fiacco TA, McCarthy KD (2008) What is the role of astrocyte calcium in neurophysiology? Neuron 59:932-946.

Agulhon C, Fiacco TA, McCarthy KD (2010) Hippocampal short- and longterm plasticity are not modulated by astrocyte $\mathrm{Ca} 2+$ signaling. Science 327:1250-1254.

Allen NJ, Lyons DA (2018) Glia as architects of central nervous system formation and function. Science 362:181-185.

Araque A, Carmignoto G, Haydon PG, Oliet SH, Robitaille R, Volterra A (2014) Gliotransmitters travel in time and space. Neuron 81:728-739. 
Attwell D, Buchan AM, Charpak S, Lauritzen M, Macvicar BA, Newman EA (2010) Glial and neuronal control of brain blood flow. Nature 468:232243.

Auer PL, Doerge RW (2010) Statistical design and analysis of RNA sequencing data. Genetics 185:405-416.

Bazargani N, Attwell D (2016) Astrocyte calcium signaling: the third wave. Nat Neurosci 19:182-189.

Benediktsson AM, Schachtele SJ, Green SH, Dailey ME (2005) Ballistic labeling and dynamic imaging of astrocytes in organotypic hippocampal slice cultures. J Neurosci Methods 141:41-53.

Bindocci E, Savtchouk I, Liaudet N, Becker D, Carriero G, Volterra A (2017) Three-dimensional $\mathrm{Ca}^{2+}$ imaging advances understanding of astrocyte biology. Science 356:eaai8185.

Brancaccio M, Patton AP, Chesham JE, Maywood ES, Hastings MH (2017) Astrocytes control circadian timekeeping in the suprachiasmatic nucleus via glutamatergic signaling. Neuron 93:1420-1435.

Chai H, Diaz-Castro B, Shigetomi E, Monte E, Octeau JC, Yu X, Cohn W, Rajendran PS, Vondriska TM, Whitelegge JP, Coppola G, Khakh BS (2017) Neural circuit-specialized astrocytes: transcriptomic, proteomic, morphological and functional evidence. Neuron 95:531-549.

Chen TW, Wardill TJ, Sun Y, Pulver SR, Renninger SL, Baohan A, Schreiter ER, Kerr RA, Orger MB, Jayaraman V, Looger LL, Svoboda K, Kim DS (2013) Ultrasensitive fluorescent proteins for imaging neuronal activity. Nature 499:295-300.

Clapham DE (2007) Calcium signaling. Cell 131:1047-1058.

Corkrum M, Covelo A, Lines J, Bellocchio L, Pisansky M, Loke K, Quintana R, Rothwell PE, Lujan R, Marsicano G, Martin ED, Thomas MJ, Kofuji P, Araque A (2020) Dopamine-evoked synaptic regulation in the nucleus accumbens requires astrocyte activity. Neuron 105:1036-1047.

Deacon RM (2013) Measuring motor coordination in mice. J Vis Exp 75: e2609.

Di Castro MA, Chuquet J, Liaudet N, Bhaukaurally K, Santello M, Bouvier D, Tiret P, Volterra A (2011) Local Ca2 + detection and modulation of synaptic release by astrocytes. Nat Neurosci 14:1276-1284.

Diaz-Castro B, Gangwani M, Yu X, Coppola G, Khakh BS (2019) Astrocyte molecular signatures in Huntington's disease. Sci Transl Med 11: eaaw8546.

Dobin A, Davis CA, Schlesinger F, Drenkow J, Zaleski C, Jha S, Batut P, Chaisson M, Gingeras TR (2013) STAR: ultrafast universal RNA-seq aligner. Bioinformatics 29:15-21.

Fiacco TA, McCarthy KD (2018) Multiple lines of evidence indicate that gliotransmission does not occur under physiological conditions. J Neurosci 38:3-13.

Fiacco TA, Agulhon C, McCarthy KD (2009) Sorting out astrocyte physiology from pharmacology. Annu Rev Pharmacol Toxicol 49:151-174.

Haim LB, Rowitch DH (2017) Functional diversity of astrocytes in neural circuit regulation. Nat Rev Neurosci 18:31-41.

Handy G, Taheri M, White JA, Borisyuk A (2017) Mathematical investigation of IP3-dependent calcium dynamics in astrocytes. J Comput Neurosci 42:257-273.

Hebenstreit D, Fang M, Gu M, Charoensawan V, van Oudenaarden A, Teichmann SA (2011) RNA sequencing reveals two major classes of gene expression levels in metazoan cells. Mol Syst Biol 7:497.

Hirbec H, Déglon N, Foo LC, Goshen I, Grutzendler J, Hangen E, Kreisel T, Linck N, Muffat J, Regio S, Rion S, Escartin C (2020) Emerging technologies to study glial cells. Glia 68:1692-1728.

Hires SA, Tian L, Looger LL (2008) Reporting neural activity with genetically encoded calcium indicators. Brain Cell Biol 36:69-86.

Howarth C (2014) The contribution of astrocytes to the regulation of cerebral blood flow. Front Neurosci 8:103.

Huang AY, Woo J, Sardar D, Lozzi B, Bosquez HN, Lin CJ, FD, Jain A, Paulucci-Holthauzen A, Deneen B (2020) Region-specific transcriptional control of astrocyte function oversees local circuit activities. Neuron 106:992-1008.e9.

Jiang R, Diaz-Castro B, Tong X, Looger LL, Khakh BS (2016) Dysfunctional calcium and glutamate signaling in striatal astrocytes from Huntington's disease model mice. J Neurosci 36:3453-3470.

Khakh BS (2019) Astrocyte-neuron interactions in the striatum: insights on identity, form, and function. Trends Neurosci 42:617-630.

Khakh BS, McCarthy KD (2015) Astrocyte calcium signaling: from observations to functions and the challenges therein. Cold Spring Harb Perspect Biol 7:a020404.
Khakh BS, Deneen B (2019) The emerging nature of astrocyte diversity. Annu Rev Neurosci 42:187-207.

Kulik A, Haentzsch A, Lückermann M, Reichelt W, Ballanyi K (1999) Neuron-glia signaling via alpha(1) adrenoceptor-mediated $\mathrm{Ca}(2+)$ release in Bergmann glial cells in situ. J Neurosci 19:8401-8408.

Kuo TH, Liu BF, Diglio C, Tsang W (1993) Regulation of the plasma membrane calcium pump gene expression by two signal transduction pathways. Arch Biochem Biophys 305:428-433.

Lee S, Yoon BE, Berglund K, Oh SJ, Park H, Shin HS, Augustine GJ, Lee CJ (2010) Channel-mediated tonic GABA release from glia. Science 330:790-796

Lee YR, Chen M, Pandolfi PP (2018) The functions and regulation of the PTEN tumour suppressor: new modes and prospects. Nat Rev Mol Cell Biol 19:547-562.

Liddelow SA, Guttenplan KA, Clarke LE, Bennett FC, Bohlen CJ, Schirmer L, Bennett ML, Münch AE, Chung WS, Peterson TC, Wilton DK, Frouin A, Napier BA, Panicker N, Kumar M, Buckwalter MS, Rowitch DH, Dawson VL, Dawson TM, Stevens B, et al. (2017) Neurotoxic reactive astrocytes are induced by activated microglia. Nature 541:481-487.

Madisen L, Garner AR, Shimaoka D, Chuong AS, Klapoetke NC, Li L, van der Bourg A, Niino Y, Egolf L, Monetti C, Gu H, Mills M, Cheng A, Tasic B, Nguyen TN, Sunkin SM, Benucci A, Nagy A, Miyawaki A, Helmchen F, et al. (2015) Transgenic mice for intersectional targeting of neural sensors and effectors with high specificity and performance. Neuron 85:942-958.

Nagai J, Rajbhandari AK, Gangwani MR, Hachisuka A, Coppola G, Masmanidis SC, Fanselow MS, Khakh BS (2019) Hyperactivity with disrupted attention by activation of an astrocyte synaptogenic cue. Cell 177:1280-1292.

Nagai J, Yu X, Papouin T, Cheong E, Freeman MR, Monk KR, Hastings MH, Haydon PG, Rowitch D, Shaham S, Khakh BS (2021) Behaviorally consequential astrocytic regulation of neural circuits. Neuron 109:576-596.

Nimmerjahn A, Bergles DE (2015) Large-scale recording of astrocyte activity. Curr Opin Neurobiol 32:95-106.

Nimmerjahn A, Mukamel EA, Schnitzer MJ (2009) Motor behavior activates Bergmann glial networks. Neuron 62:400-412.

Octeau JC, Chai H, Jiang R, Bonanno SL, Martin KC, Khakh BS (2018) An optical neuron-astrocyte proximity assay at synaptic distance scales. Neuron 98:49-66.

Oliveira JF, Sardinha VM, Guerra-Gomes S, Araque A, Sousa N (2015) Do stars govern our actions? Astrocyte involvement in rodent behavior. Trends Neurosci 38:535-549.

Otsu Y, Couchman K, Lyons DG, Collot M, Agarwal A, Mallet JM, Pfrieger FW, Bergles DE, Charpak S (2015) Calcium dynamics in astrocyte processes during neurovascular coupling. Nat Neurosci 18:210-218.

Pestana F, Edwards-Faret G, Belgard TG, Martirosyan A, Holt MG (2020) No longer underappreciated: the emerging concept of astrocyte heterogeneity in neuroscience. Brain Sci 10:168.

Petravicz J, Fiacco TA, McCarthy KD (2008) Loss of IP3 receptor-dependent $\mathrm{Ca} 2+$ increases in hippocampal astrocytes does not affect baseline CA1 pyramidal neuron synaptic activity. J Neurosci 28:4967-4973.

Petravicz J, Boyt KM, McCarthy KD (2014) Astrocyte IP3R2-dependent Ca $(2+)$ signaling is not a major modulator of neuronal pathways governing behavior. Front Behav Neurosci 8:384.

Piet R, Jahr CE (2007) Glutamatergic and purinergic receptor-mediated calcium transients in Bergmann glial cells. J Neurosci 27:4027-4035.

Porter JT, McCarthy KD (1997) Astrocytic neurotransmitter receptors in situ and in vivo. Prog Neurobiol 51:439-455.

Robinson MD, McCarthy DJ, Smyth GK (2010) edgeR: a bioconductor package for differential expression analysis of digital gene expression data. Bioinformatics 26:139-140.

Roth BJ, Yagodin SV, Holtzclaw L, Russell JT (1995) A mathematical model of agonist-induced propagation of calcium waves in astrocytes. Cell Calcium 17:53-64.

Rungta RL, Bernier L-P, Dissing-Olesen L, Groten CJ, LeDue JM, Ko R, Drissler S, MacVicar BA (2016) Ca2 + transients in astrocyte fine processes occur via Ca2+ influx in adult mouse hippocampus. Glia 64:20932103.

Russell JT (2011) Imaging calcium signals in vivo: a powerful tool in physiology and pharmacology. Br J Pharmacol 163:1605-1625.

Saunders A, Macosko EZ, Wysoker A, Goldman M, Krienen FM, de Rivera H, Bien E, Baum M, Bortolin L, Wang S, Goeva A, Nemesh J, Kamitaki 
N, Brumbaugh S, Kulp D, McCarroll SA (2018) Molecular diversity and specializations among the cells of the adult mouse brain. Cell 174:1015-1030.

Savtchenko LP, Bard L, Jensen TP, Reynolds JP, Kraev I, Medvedev N, Stewart MG, Henneberger C, Rusakov DA (2018) Disentangling astroglial physiology with a realistic cell model in silico. Nat Commun 9:3554.

Savtchouk I, Volterra A (2018) Gliotransmission: beyond black-and-white. J Neurosci 38:14-25.

Shigetomi E, Bushong EA, Haustein MD, Tong X, Jackson-Weaver O, Kracun S, Xu J, Sofroniew MV, Ellisman MH, Khakh BS (2013) Imaging calcium microdomains within entire astrocyte territories and endfeet with GCaMPs expressed using adeno-associated viruses. J Gen Physiol 141:633-647.

Shigetomi E, Patel S, Khakh BS (2016) Probing the complexities of astrocyte calcium signaling. Trends Cell Biol 26:300-312.

Srinivasan R, Huang BS, Venugopal S, Johnston AD, Chai H, Zeng H, Golshani P, Khakh BS (2015) Ca(2+) signaling in astrocytes from Ip3r2 $(-/)$ mice in brain slices and during startle responses in vivo. Nat Neurosci 18:708-717.

Srinivasan R, Lu TY, Chai H, Xu J, Huang BS, Golshani P, Coppola G, Khakh BS (2016) New transgenic mouse lines for selectively targeting astrocytes and studying calcium signals in astrocyte processes in situ and in vivo. Neuron 92:1181-1195.

Taheri M, Handy G, Borisyuk A, White JA (2017) Diversity of evoked astrocyte $\mathrm{Ca} 2+$ dynamics quantified through experimental measurements and mathematical modeling. Front Syst Neurosci 11:79.

Tong X, Ao Y, Faas GC, Nwaobi SE, Xu J, Haustein MD, Anderson MA, Mody I, Olsen ML, Sofroniew MV, Khakh BS (2014) Astrocyte Kir4.1 ion channel deficits contribute to neuronal dysfunction in Huntington's disease model mice. Nat Neurosci 17:694-703.

Uhlen M, Zhang C, Lee S, Sjöstedt E, Fagerberg L, Bidkhori G, Benfeitas R, Arif M, Liu Z, Edfors F, Sanli K, von Feilitzen K, Oksvold P, Lundberg E, Hober S, Nilsson P, Mattsson J, Schwenk JM, Brunnström H, Glimelius B, et al. (2017) A pathology atlas of the human cancer transcriptome. Science 357:eaan2507.

Ung K, Tepe B, Pekarek B, Arenkiel BR, Deneen B (2020) Parallel astrocyte calcium signaling modulates olfactory bulb responses. J Neurosci Res 98:1605-1618.
Verkhratsky A, Nedergaard M (2018) Physiology of astroglia. Physiol Rev 98:239-389.

Voleti V, Patel KB, Li W, Campos CP, Bharadwaj S, Yu H, Ford C, Casper MJ, Yan RW, Liang W, Wen C, Kimura KD, Targoff KL, Hillman EMC (2019) Real-time volumetric microscopy of in vivo dynamics and largescale samples with SCAPE 2.0. Nat Methods 16:1054-1062.

Volterra A, Liaudet N, Savtchouk I (2014) Astrocyte $\mathrm{Ca}(2)(+)$ signalling: an unexpected complexity. Nat Rev Neurosci 15:327-335.

Wang Y, DelRosso NV, Vaidyanathan TV, Cahill MK, Reitman ME, Pittolo S, Mi X, Yu G, Poskanzer KE (2019) Accurate quantification of astrocyte and neurotransmitter fluorescence dynamics for single-cell and population-level physiology. Nat Neurosci 22:1936-1944.

Watanabe M (2002) Glial processes are glued to synapses via $\mathrm{Ca}(2+)$-permeable glutamate receptors. Trends Neurosci 25:5-6.

Wolf FA, Angerer P, Theis FJ (2018) SCANPY: large-scale single-cell gene expression data analysis. Genome Biol 19:15

Xie Y, Wang T, Sun GY, Ding S (2010) Specific disruption of astrocytic $\mathrm{Ca} 2+$ signaling pathway in vivo by adeno-associated viral transduction. Neuroscience 170:992-1003.

Xu CS, Hayworth KJ, Lu Z, Grob P, Hassan AM, García-Cerdán JG, Niyogi KK, Nogales E, Weinberg RJ, Hess HF (2017) Enhanced FIB-SEM systems for large-volume 3D imaging. Elife 6:e25916.

Yu X, Taylor AMW, Nagai J, Golshani P, Evans CJ, Coppola G, Khakh BS (2018) Reducing astrocyte calcium signaling in vivo alters striatal microcircuits and causes repetitive behavior. Neuron 99:1170-1187.

Yu X, Nagai J, Khakh BS (2020a) Improved tools to study astrocytes. Nat Rev Neurosci 21:121-138.

Yu X, Nagai J, Marti-Solano M, Soto JS, Coppola G, Babu MM, Khakh BS (2020b) Context-specific striatal astrocyte molecular responses are phenotypically exploitable. Neuron 108:1146-1162.e10.

Zeisel A, Hochgerner H, Lönnerberg P, Johnsson A, Memic F, van der Zwan J, Haring M, Braun E, Borm LE, La Manno G, Codeluppi S, Furlan A, Lee K, Skene N, Harris KD, Hjerling-Leffler J, Arenas E, Ernfors P, Marklund U, Linnarsson S (2018) Molecular architecture of the mouse nervous system. Cell 174:999-1014.e22 